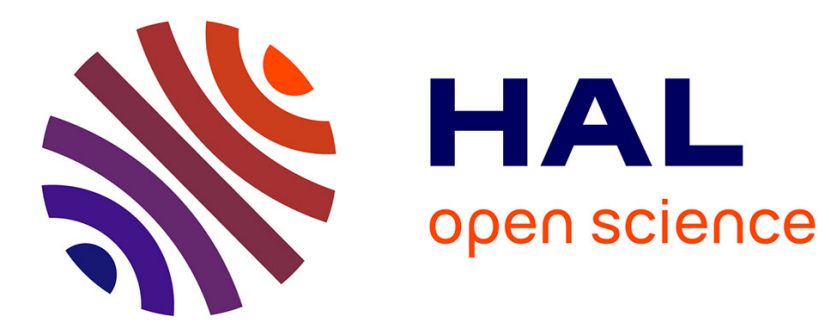

\title{
Analysis of added mass in cavitating flow
}

Mustapha Benaouicha, Jacques Andre Astolfi

\section{To cite this version:}

Mustapha Benaouicha, Jacques Andre Astolfi. Analysis of added mass in cavitating flow. Journal of Fluids and Structures, 2012, 31, pp.30-48. 10.1016/j.jfluidstructs.2012.03.003 . hal-01081223

\section{HAL Id: hal-01081223 https://hal.science/hal-01081223}

Submitted on 7 Nov 2014

HAL is a multi-disciplinary open access archive for the deposit and dissemination of scientific research documents, whether they are published or not. The documents may come from teaching and research institutions in France or abroad, or from public or private research centers.
L'archive ouverte pluridisciplinaire HAL, est destinée au dépôt et à la diffusion de documents scientifiques de niveau recherche, publiés ou non, émanant des établissements d'enseignement et de recherche français ou étrangers, des laboratoires publics ou privés. 


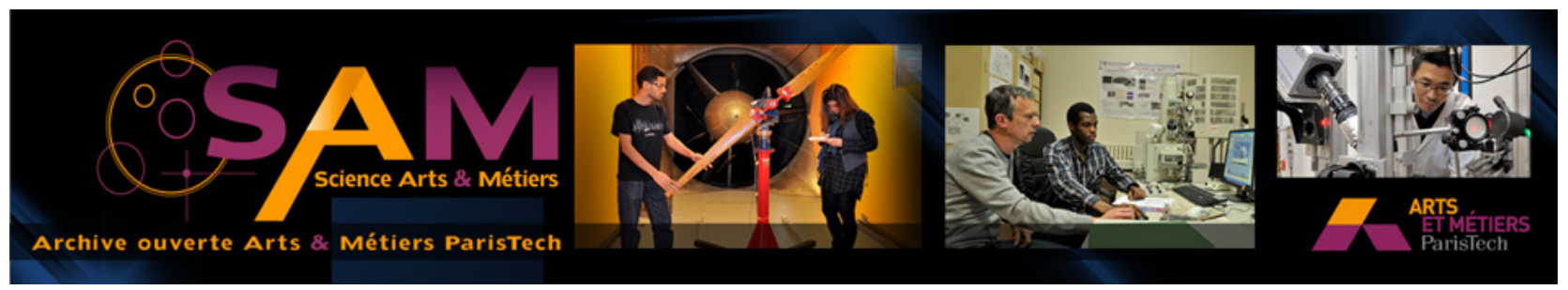

Science Arts \& Métiers (SAM)

is an open access repository that collects the work of Arts et Métiers ParisTech researchers and makes it freely available over the web where possible.

This is an author-deposited version published in: http://sam.ensam.eu Handle ID: .http://hdl.handle.net/10985/8836

\section{To cite this version :}

Mustapha BENAOUICHA, Jacques Andre ASTOLFI - Analysis of added mass in cavitating flow Journal of Fluid and Structure - Vol. 31, p.30-48 - 2012 


\title{
Analysis of added mass in cavitating flow
}

\author{
Mustapha Benaouicha*, Jacques-André Astolfi \\ French Naval Academy Research Institute (IRENav)/ENGEP, CC600, 29240 Brest Cedex 09, France
}

Keywords:

Fluid-structure interaction

Cavitation induced vibrations

Added mass

\begin{abstract}
A B S T R A C T
The paper addresses a theoretical study of the added mass effect in cavitating flow. The cavitation is considered to induce a strong time-space variation of the fluid density at the interface between an inviscid fluid and a three-degree-of-freedom rigid section. The coupled problem is then simplified to a Laplace equation written for the pressure with a boundary condition at the fluid-structure interface depending on the acceleration, the velocity of the structure and on the rate of change of flow density. It is shown that contrary to the homogeneous flow, the added mass operator is not symmetrical and depends on the flow through fluid density variation. The added mass coefficients decrease as the cavitation increases which should induce an increase of the natural structural frequencies. The model shows also an added damping operator related to the rate of change of flow density. Added damping coefficients are found to be positive or negative according to the rate of change of the fluid density, indicating the possibility of instability development between flexible structures and unsteady cavitating flows.
\end{abstract}

\section{Introduction}

Cavitation occurs in liquid flows if pressure locally drops below the vapor pressure (Brennen, 1995; Caupin and Herbert, 2006). This can be observed in hydraulic systems like pumps, injectors, marine propellers or hydrofoils at high speed. Undesirable features of cavitation are performance losses, erosion, noises and vibrations that can seriously damage the systems.

Various cavitation patterns can occur in a flow. Sheet cavitation is currently encountered on blades of hydraulic systems. It corresponds to the situation for which a vapor cavity is attached at the leading edge of the blade and extends over a distance on the surface, namely the cavity length. If the cavity length is smaller than the blade chord, the sheet cavitation is also named partial cavitation.

Conversely supercavitation corresponds to situation for which the vapor cavity extends far beyond the foil trailing edge. Sheet cavitation is unsteady by nature. For relatively small cavity lengths, the unsteadiness is localized in the rear part of the cavity, while for larger cavity lengths the vaporized area becomes unstable with a periodical break-off of the cavity and the periodical shedding of large bubble clusters (Leroux et al., 2005). This configuration, usually called "cloud cavitation", generates periodical wall pressure fluctuations, high levels of vibration, and acoustic radiation. In that case, a part of the foil surface passes periodically from liquid to vapor and experiences a strong periodical variation of the fluid density from about 1 for the vapor to 1000 for the liquid.

\footnotetext{
* Corresponding author. Tel.: +33298234 142; fax: +33298233857.

E-mail addresses: mustapha.benaouicha@ecole-navale.fr (M. Benaouicha), jacques-andre.astolfi@ecole-navale.fr (J.-A. Astolfi).
} 
Computational modeling of cavitation has been pursued for years. An overview of selected studies is presented by Wang et al. (2001). Studies dealing with cavitation modeling through the computation of the Navier-Stokes equations have emerged in the past two decades (Chen and Heister, 1995; Goncalvès, 2011; Kubota et al., 1992; Leroux et al., 2005; Shukla et al., 2010; Ventikos and Tzabiras, 2000) but the prediction of such non-stationary, turbulent and locally compressible two-phase flows remains a challenge.

Numerous authors have noted cavitation impact on fluid-structure interaction of various devices, especially propeller blades and hydrofoils, but estimations of this impact are not frequent (Amromin and Kovinskaya, 2000; Ross et al., 2008, 2009; Young, 2007, 2008).

For example, Kopriva et al. (2007) described approximately a threefold difference in the first structural resonance frequencies determined for a foil in vacuum and in cavitating flow, but the added mass effect (convenient for engineer analysis) was not separated. Moreover, experimental studies recently carried out in the Research Institute of French Naval Academy have shown that the modal response of the structure could be modified in the presence of cavitation (Benaouicha et al., 2010). This modification can be attributed to the presence in flow of non-stationary liquid-vapor mixture with both strong density variations and sound celerity at the fluid-structure interface, significantly modifying the coupling conditions and the induced inertial effects.

Our interest in the present work is the study of the impact of such variations in cavitation-structure interactions. One point of interest is a better understanding of the added mass operator. A theoretical model is proposed and resolved by finite element method. The model has the advantage of being a generalization of homogeneous flow model (Axisa and Antunes, 2007) to non-homogeneous flows. Comparisons with results obtained from a potential flow method in homogeneous flow were carried out (Blevins, 1995; Han and Xu, 1996; Korotkin, 2009; Newman, 1977). Nonhomogeneous flow conditions are then considered. The model can be applied to compute the added mass operator for any geometry. In this paper, symmetrical (rectangle) and asymmetrical (cambered hydrofoil) geometries are tested.

\section{Coupled problem and added mass concept}

The general scenario of a coupled problem in fluid-structure interaction is that the flow induces structure vibration and that the displacements of the fluid-structure interface locally modifies the flow. A $2 d$-configuration of this coupled problem is studied by considering a three-degree-of-freedom rigid body motion (translation and rotation) of a $2 d$-section in Cartesian coordinates (Fig. 1). The fluid domain $\Omega_{F}$ is supposed large compared to the structure $\Omega_{S}$, so that thereafter it is considered as infinite. $\Gamma_{I}$ stands for the inlet section, $\Gamma_{O}$ the outlet section of the flow, $\Gamma_{W}$ the wall (fixed boundary) and $\Gamma_{F S}$ the fluid-structure interface. $\mathbf{n}$ denotes the unit normal vector at $\partial \Omega_{F}=\Gamma_{I} \cup \Gamma_{O} \cup \Gamma_{W} \cup \Gamma_{F S}$ pointing out of $\Omega_{F}$.

The velocity $\dot{\xi}$ and acceleration $\ddot{\xi}$ of a point $A(x, y)$ on the interface $\Gamma_{F S}$ are defined as

$$
\dot{\xi}(A, t)=\dot{\xi}(O, t)+\omega \wedge \mathbf{r}=\dot{\xi}_{1} \mathbf{i}+\dot{\xi}_{2} \mathbf{j}
$$

and

$$
\ddot{\xi}(A, t)=\ddot{\xi}(O, t)+\dot{\omega} \wedge \mathbf{r}+\omega \wedge(\omega \wedge \mathbf{r})=\ddot{\xi}_{1} \mathbf{i}+\ddot{\xi}_{2} \mathbf{j},
$$

$O\left(x_{0}, y_{0}\right)$ being the center of rotation of the body $(\mathbf{r}=\overrightarrow{A O}), \omega=\omega \mathbf{k}$ the angular velocity and $\dot{\omega}=\dot{\omega} \mathbf{k}$ the angular acceleration $(\mathbf{k}=\mathbf{i} \wedge \mathbf{j})$.

The body displacement $\xi$, velocity $\dot{\xi}$ and acceleration $\ddot{\xi}$ are functions of fluid forces and moments, acting on the solid surface $\Gamma_{F S}$. The fluid loading can be decomposed to forces of inertial nature, which can be computed assuming that the fluid is ideal, and forces that are related to viscosity. The forces of inertial nature can be expressed in terms of added mass (Korotkin, 2009). Furthermore, it is often required to separate the added mass forces from the viscous forces (Ackermann et al., 1964; Brennen, 1995; McConnell and Young, 1965).

The aim of this study being the analysis of added mass operator, it then can be assumed that the viscosity effects are neglected.

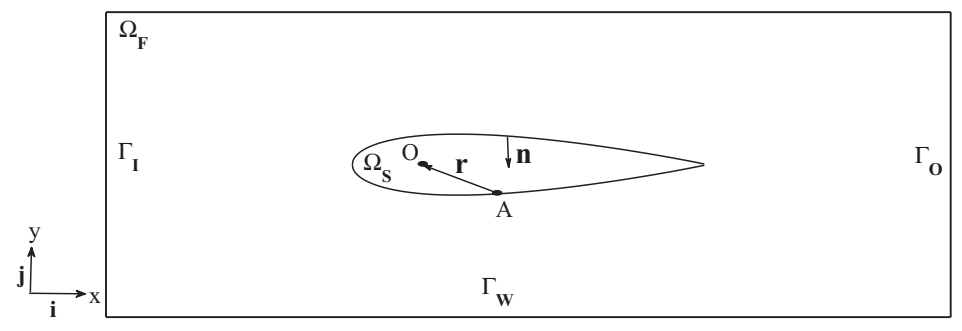

Fig. 1. Fluid and solid domains. 
Let us consider the following conservation equations describing the ideal fluid flow in $\Omega_{F}$ :

$$
\left\{\begin{array}{lll}
\frac{\partial \rho}{\partial t}+\nabla \cdot(\rho u)=0 & \text { on } \Omega_{F}, & \text { (a) } \\
\frac{\partial(\rho u)}{\partial t}+\nabla \cdot(\rho u \otimes u)=-\nabla p & \text { on } \Omega_{F}, & \text { (b) } \\
u=u_{I} & \text { on } \Gamma_{I}, & \text { (c) } \\
-p \mathbf{n}=0 & \text { on } \Gamma_{O}, & \text { (d) } \\
u \cdot \mathbf{n}=0 & \text { on } \Gamma_{W}, & \text { (e) } \\
u \cdot \mathbf{n}=\dot{\xi} \cdot \mathbf{n} & \text { on } \Gamma_{F S}, & \text { (f) }
\end{array}\right.
$$

where $\rho, u$ and $p$ are respectively the fluid mass density, the velocity field and the pressure field in the fluid.

\subsection{Added mass in homogeneous flow}

In inviscid and homogeneous single phase flow ( $\rho$ assumed constant in time and space), potential theory (Newman, 1977; Wang, 2008) is often used to predict the forces and moments acting on the body due to dynamic pressure of the fluid. A rigid body motion in infinite fluid domain is considered. The fluid is assumed to be incompressible and irrotational.

Introducing a velocity potential $\phi$, such that $u=\nabla \phi$, and ignoring the gravity effect, the following Laplace and Bernoulli equations are derived from Euler equations (3),

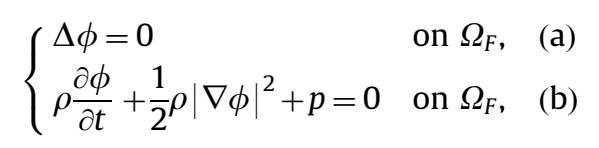

with the moving boundary condition on $\Gamma_{F S}$,

$$
\nabla \phi \cdot \mathbf{n}=\dot{\xi} \cdot \mathbf{n}
$$

and infinity condition,

$$
\left.\phi(t, \mathbf{r})\right|_{\mathbf{r} \rightarrow \infty}=0, \quad \forall t \in \mathbf{R}^{+} .
$$

The total force and moment acting on the rigid body are expressed as

$$
\begin{aligned}
& \mathbf{F}(t)=-\rho \frac{d}{d t} \int_{\Gamma_{\mathrm{FS}}} \phi \mathbf{n} d s, \\
& \mathcal{M}(t)=-\rho \frac{d}{d t} \int_{\Gamma_{\mathrm{FS}}} \phi(\mathbf{r} \times \mathbf{n}) d s .
\end{aligned}
$$

The added mass tensor is then defined as

$$
m_{i j}^{a}=\rho \int_{\Gamma_{F S}} \phi_{j} \frac{\partial \phi_{i}}{\partial \mathbf{n}} d s=\rho \int_{\Omega_{F}} \nabla \phi_{i} \cdot \nabla \phi_{j} d v,
$$

where $\phi_{i}$ represents the velocity potential due to a body motion with unit velocity in the $i$ th mode (Newman, 1977; Wang, 2008).

One convenient feature of the added mass tensor is the symmetry ( $m_{i j}^{a}=m_{j i}^{a}$ ). It is also easy to see that the added mass coefficients depend only on the body shape.

\section{Added mass operator in non-homogeneous flow}

In that case, assumptions required in potential theory are not hold and added mass operator cannot be computed directly from Eq. (9). A cavitating flow can be considered as an incompressible two-phase flow with large variations of density from liquid to vapor resulting of hydrodynamic pressure variation (Coutier-Delgosha et al., 2007). Then, a set of equations must be derived to take into account the variations of two-phase fluid density at the fluid-structure interface.

\subsection{Linearized flow equations}

Let us assume that $u=\tilde{u}+u^{\prime}$ and $p=\tilde{p}+p^{\prime}$ where $\tilde{u}$ and $\tilde{p}$ are the velocity and pressure fields of the fluid flow around a non-vibrating structure; and $u^{\prime}$ and $p^{\prime}$ are the velocity and pressure fields generated by the vibration of the structure. The 
variations of $\rho$ due to the vibration of the structure are assumed negligible. Eqs. (3) are then written as

$$
\left\{\begin{array}{l}
\frac{\partial \rho}{\partial t}+\nabla \cdot(\rho \tilde{u})+\nabla \cdot\left(\rho u^{\prime}\right)=0 \\
\frac{\partial(\rho \tilde{u})}{\partial t}+\nabla \cdot(\rho \tilde{u} \otimes \tilde{u})+\nabla \tilde{p}+ \\
\frac{\partial\left(\rho u^{\prime}\right)}{\partial t}+\nabla \cdot\left(\rho u^{\prime} \otimes u^{\prime}\right)+\nabla p^{\prime}=-\nabla \cdot\left(\rho\left[\left(\tilde{u} \otimes u^{\prime}\right)+\left(u^{\prime} \otimes \tilde{u}\right)\right]\right)
\end{array}\right.
$$

In the case of unbounded fluid domain, $u^{\prime}$ vanishes far from the mobile fluid-structure interface. Then the infinity condition, $u^{\prime}=0$ on $\partial \Omega_{F} \backslash \Gamma_{F S}$, holds. The following separate boundary conditions are therefore considered

$$
\left\{\begin{array}{lll}
\tilde{u}=u_{I} \text { and } u^{\prime}=0 & \text { on } \Gamma_{I}, & \text { (a) } \\
\tilde{p} \mathbf{n}=0 \text { and } u^{\prime}=0 & \text { on } \Gamma_{O}, & \text { (b) } \\
\tilde{u} \cdot \mathbf{n}=0 \text { and } u^{\prime}=0 & \text { on } \Gamma_{W}, & \text { (c) } \\
\tilde{u} \cdot \mathbf{n}=0 \text { and } u^{\prime} \cdot \mathbf{n}=\dot{\xi} \cdot \mathbf{n} & \text { on } \Gamma_{F S}, & \text { (d) }
\end{array}\right.
$$

$u^{\prime}$ is supposed to be small so that $u^{\prime} \otimes u^{\prime}$ can be neglected. Let us notice that if $\tilde{u}=0$ on $\Gamma_{F S}$ (which is the case in viscous fluid flow) then the average value over $\Omega_{F}$ of the right-hand side of Eq. (10c) is null. Indeed, by using the Ostrogradski theorem and boundary conditions (11), we have

$$
\int_{\Omega_{\mathrm{F}}} \nabla \cdot\left(\rho\left[\left(\tilde{u} \otimes u^{\prime}\right)+\left(u^{\prime} \otimes \tilde{u}\right)\right]\right) d V=\int_{\partial \Omega_{\mathrm{F}}}\left(\rho\left[\left(\tilde{u} \otimes u^{\prime}\right)+\left(u^{\prime} \otimes \tilde{u}\right)\right]\right) \cdot \mathbf{n} d S=0 .
$$

If we assume that $u$ and $u^{\prime}$ are uncorrelated, it can be assumed that this coupling term is negligible in $\Omega_{F}$. The problem (10) can then be split into two separate problems

$$
\left\{\begin{array}{lll}
\frac{\partial \rho}{\partial t}+\nabla \cdot(\rho \tilde{u})=0 & \text { on } \Omega_{F}, & \text { (a) } \\
\frac{\partial(\rho \tilde{u})}{\partial t}+\nabla \cdot(\rho \tilde{u} \otimes \tilde{u})=-\nabla \tilde{p} & \text { on } \Omega_{F}, & \text { (b) } \\
\tilde{u}=\bar{u}_{I} & \text { on } \Gamma_{I}, & \text { (c) } \\
\tilde{p} \mathbf{n}=0 & \text { on } \Gamma_{O}, & \text { (d) } \\
\tilde{u} \cdot \mathbf{n}=0 & \text { on } \Gamma_{W} \cup \Gamma_{F S}, & \text { (e) }
\end{array}\right.
$$

where $\tilde{u}$ refers to the fluid velocity around the non-vibrating structure, and

$$
\left\{\begin{array}{lll}
\nabla \cdot\left(\rho u^{\prime}\right)=0 & \text { on } \Omega_{F}, & \text { (a) } \\
\frac{\partial\left(\rho u^{\prime}\right)}{\partial t}+\nabla p^{\prime}=0 & \text { on } \Omega_{F}, & \text { (b) } \\
u^{\prime} \cdot \mathbf{n}=\dot{\xi} \cdot \mathbf{n} & \text { on } \Gamma_{F S}, & \text { (c) } \\
u^{\prime}=0 & \text { on } \partial \Omega_{F} \backslash \Gamma_{F S}, & \text { (d) }
\end{array}\right.
$$

where $u^{\prime}$ refers to the fluid velocity induced by the structure vibrations $\dot{\xi}$.

Actually, the two problems can be seen as being coupled. In fact, the fluid-structure interface velocity $\dot{\xi}$ is a function of $p=\tilde{p}+p^{\prime}$. It is thus function of $\tilde{u}, \tilde{p}, u^{\prime}$ and $p^{\prime}$. The objective being the analysis of added mass operator, Eq. (13) has to be solved.

By taking the divergence of Eq. (13b) and considering Eq. (13a), problem (13) can be rewritten, after the projection of Eq. (13b) on the outgoing normal vector $\mathbf{n}$ of the fluid domain at $\Gamma_{F S}$, in the following form:

$$
\left\{\begin{array}{lll}
\Delta p^{\prime}=0 & \text { on } \Omega_{F}, \\
\frac{\partial\left(\rho u^{\prime}\right)}{\partial t} \cdot \mathbf{n}=-\nabla p^{\prime} \cdot \mathbf{n} & \text { on } \Gamma_{F S}, & \text { (a) } \\
p^{\prime}=0 & \text { on } \partial \Omega_{F} \backslash \Gamma_{F S}, & \text { (c) }
\end{array}\right.
$$

the left term of Eq. (14b) can be developed, on $\Gamma_{\mathrm{FS}}$ and for $t \in[0,+\infty[$, as follows:

$$
\frac{\partial\left(\rho u^{\prime}\right)}{\partial t} \cdot \mathbf{n}=\left(\rho \frac{\partial u^{\prime}}{\partial t}+\frac{\partial \rho}{\partial t} u^{\prime}\right) \cdot \mathbf{n},
$$

taking account of the boundary condition (13c), we have

$$
\frac{\partial\left(\rho u^{\prime}\right)}{\partial t} \cdot \mathbf{n}=\rho \ddot{\xi} \cdot \mathbf{n}+\frac{\partial \rho}{\partial t} \dot{\xi} \cdot \mathbf{n} \quad \text { on } \Gamma_{F S}
$$


Finally, Eq. (14) becomes

$$
\left\{\begin{array}{lll}
\Delta p^{\prime}=0 & \text { on } \Omega_{F}, & \text { (a) } \\
\nabla p^{\prime} \cdot \mathbf{n}=-\rho \ddot{\xi} \cdot \mathbf{n}-\frac{\partial \rho_{\dot{m}}}{\partial t} \cdot \mathbf{n} & \text { on } \Gamma_{F S}, & \text { (b) } \\
p^{\prime}=0 & \text { on } \partial \Omega_{F} \backslash \Gamma_{F S .} & \text { (c) }
\end{array}\right.
$$

Where boundary condition (15b) depends on the acceleration and the velocity of the structure together with the rate of change of the density at the fluid-structure interface. Due to the linearity, the superposition principle holds and the solution of (15) can be expressed as $p^{\prime}=p_{1}+p_{2}$, where $p_{1}$ and $p_{2}$ are respectively the solutions of the following problems:

$$
\left\{\begin{array}{lll}
\Delta p_{1}=0 & \text { on } \Omega_{F}, & \text { (a) } \\
\nabla p_{1} \cdot \mathbf{n}=-\rho \ddot{\xi} \cdot \mathbf{n} & \text { on } \Gamma_{F S}, & \text { (b) } \\
p_{1}=0 & \text { on } \partial \Omega_{F} \backslash \Gamma_{F S} & \text { (c) }
\end{array}\right.
$$

and

$$
\left\{\begin{array}{lll}
\Delta p_{2}=0 & \text { on } \Omega_{F}, & \text { (a) } \\
\nabla p_{2} \cdot \mathbf{n}=-\frac{\partial \rho_{\dot{m}}}{\partial t} \cdot \mathbf{n} & \text { on } \Gamma_{F S}, & \text { (b) } \\
p_{2}=0 & \text { on } \partial \Omega_{F} \backslash \Gamma_{F S} . & \text { (c) }
\end{array}\right.
$$

The solution $p_{1}$ of Eq. (16) represents the inertial effect of the fluid on the structure, as it is proportional to the acceleration $\ddot{\xi}$ of the structure. The solution $p_{2}$ of Eq. (17) can be interpreted as time and spatial variations effect of fluid density on the flow-structure interface. It can be related to the damping effect as it is dependent on the velocity $\dot{\xi}$ of the structure.

\subsection{Inertial effect}

Because of linearity, the solution $p_{1}$ of problem (16) is proportional to the normal acceleration $\ddot{\xi} \cdot \mathbf{n}$ of $\Gamma_{F S}$; therefore the structure loading due to the pressure field $p_{1}$ is a force $\mathbf{F}(t)=F_{1} \mathbf{i}+F_{2} \mathbf{j}$ and a moment $\mathcal{M}(t)=\mathcal{M}(t) \mathbf{k}$ which are also proportional to the acceleration of $\Gamma_{F S}$. They are represented by the following integrals:

$$
\mathbf{F}(t)=-\int_{\Gamma_{\mathrm{FS}}} p_{1} \mathbf{n} d s
$$

and

$$
\mathcal{M}(t)=-\int_{\Gamma_{F S}} p_{1}(\mathbf{r} \wedge \mathbf{n}) d s
$$

they can be written as

$$
\left\{\begin{array}{l}
F_{1}(t)=-m_{11}^{a} \ddot{X}_{1}-m_{12}^{a} \ddot{X}_{2}-m_{13}^{a} \ddot{X}_{3}, \\
F_{2}(t)=-m_{21}^{a} \ddot{X}_{1}-m_{22}^{a} \ddot{X}_{2}-m_{23}^{a} \ddot{X}_{3}, \\
\mathcal{M}(t)=-m_{31}^{a} \ddot{X}_{1}-m_{32}^{a} \ddot{X}_{2}-m_{33}^{a} \ddot{X}_{3},
\end{array}\right.
$$

where $\ddot{X}_{1}(t)=\ddot{\xi}_{1}(O, t)$ and $\ddot{X}_{2}=\ddot{\xi}_{2}(O, t)$ represent the translational (surge and heave) acceleration according to the $2 d-$ coordinates axis, $\ddot{X}_{3}(t)=\dot{\omega}(t)$ the angular (pitch) acceleration and $\left(m_{i j}^{a}\right)_{i, j=1,2,3}$ are the added mass coefficients.

The matrix $\mathbf{M}^{(a)}$ such that

$$
\mathcal{F}(t)=-\mathbf{M}^{(a)} \ddot{X}=-\left(m_{i j}^{a} \ddot{X}_{j}\right)_{i=1,2,3},
$$

where $\mathcal{F}=\left(F_{1}, F_{2}, \mathcal{M}\right)^{T}$ and $\ddot{X}=\left(\ddot{X}_{i}\right)_{i=1,2,3}$, is the added mass- and moment-matrix.

Eq. (21) implies that acceleration in the i-direction $\left(\ddot{X}_{1} \neq 0, \ddot{X}_{2}=0, \ddot{X}_{3}=0\right.$ ) of a general cross section will induce not only an added mass force in the $\mathbf{i}$-direction, but also a force in the $\mathbf{j}$-direction and a moment about the center of rotation (taken as origin). If the cross section is symmetrical about the $\mathbf{i}$ - and $\mathbf{j}$-axes, the added mass forces are not coupled, and only three independent diagonal terms ( $m_{i j}^{a}, i=1,2,3$ ) remain in the added mass matrix (Blevins, 1995).

It can be noted that in $2 \mathrm{D}$ case, the dimension of $F_{1}$ and $F_{2}$ is a force per unit length $(\mathrm{N} / \mathrm{m})$ and the dimension of $\mathcal{M}$ is a moment per unit length $(\mathrm{N} \mathrm{m} / \mathrm{m}=\mathrm{N})$. Thus the dimensions of matrix $\mathbf{M}^{(a)}$ coefficients are: mass per unit length (kg/m) for $\left(m_{i j}^{a}\right)_{i, j=1,2}$, moment of inertia per unit length $\left(\mathrm{kg} \mathrm{m}^{2} / \mathrm{m}=\mathrm{kg} \mathrm{m}\right)$ for $m_{33}^{a}$ and mass $(\mathrm{kg})$ for $\left(m_{i 3}^{a} \text { and } m_{3 i}^{a}\right)_{i=1,2}$.

\subsection{Damping effect}

The same arguments as in the previous section can be used to argue that the solution $p_{2}$ of problem (17) is proportional to the normal velocity $\dot{\xi} \cdot \mathbf{n}$ of $\Gamma_{F S}$; therefore the structure loading due to the pressure field $p_{2}$ is a force $\mathbf{F}^{*}(t)=F_{1}^{*} \mathbf{i}+F_{2}^{*} \mathbf{j}$ and a moment $\mathcal{M}^{*}(t)=\mathcal{M}^{*}(t) \mathbf{k}$ which are also proportional to the velocity of $\Gamma_{F S}$. Consequently, the load acts as a damping force (and moment) due to time and spatial variations of fluid density. 
The same approach as (3.2) leads to the following relations:

$$
\left\{\begin{array}{l}
F_{1}^{*}(t)=-d_{11}^{a} \dot{X}_{1}-d_{12}^{a} \dot{X}_{2}-d_{13}^{a} \dot{X}_{3}, \\
F_{2}^{*}(t)=-d_{21}^{a} \dot{X}_{1}-d_{22}^{a} \dot{X}_{2}-d_{23}^{a} \dot{X}_{3}, \\
\mathcal{M}^{*}(t)=-d_{31}^{a} \dot{X}_{1}-d_{32}^{a} \dot{X}_{2}-d_{33}^{a} \dot{X}_{3},
\end{array}\right.
$$

where $\dot{X}_{1}(t)=\dot{\xi}_{1}(O, t)$ and $\dot{X}_{2}=\dot{\xi}_{2}(O, t)$ represent the translational (surge and heave) velocity according to the $2 d$ coordinate axis, $\dot{X}_{3}(t)=\omega(t)$ the angular (pitch) velocity and $\left(d_{i j}^{a}\right)_{i, j=1,2,3}$ are the proportional coefficients.

The matrix $\mathbf{D}^{(a)}$ such that

$$
\mathcal{F}^{*}(t)=-\mathbf{D}^{(a)} \dot{X}=-\left(d_{i j}^{a} \dot{X}_{j}\right)_{i=1,2,3},
$$

where $\mathcal{F}^{*}=\left(F_{1}^{*}, F_{2}^{*}, \mathcal{M}^{*}\right)^{T}$ and $\dot{X}=\left(\dot{X}_{i}\right)_{i=1,2,3}$ is the added damping matrix due to the fluid density variations.

It can be noted that in 2D case, the dimension of $F_{1}^{*}$ and $F_{2}^{*}$ is a force per unit length $(\mathrm{N} / \mathrm{m})$ and the dimension of $\mathcal{M}^{*}$ is a moment per unit length $(\mathrm{N} \mathrm{m} / \mathrm{m}=\mathrm{N})$. Thus the dimensions of matrix $\mathbf{D}^{(a)}$ coefficients are: damping per unit length $(\mathrm{kg} / \mathrm{s} \mathrm{m})$ for $\left(d_{i j}^{a}\right)_{i, j=1,2}$, angular dumping per unit length $\left(\mathrm{kg} \mathrm{m}^{2} / \mathrm{s} \mathrm{m}=\mathrm{kg} \mathrm{m} / \mathrm{s}\right)$ for $d_{33}^{a}$ and mass per unit time $(\mathrm{kg} / \mathrm{s})$ for $\left(d_{i 3}^{a}\right.$ and $\left.d_{3 i}^{a}\right)_{i=1,2}$.

\section{Numerical resolution in homogeneous flow}

In this section, the finite element code CASTEM (CEA, 2009) is used to solve Eq. (16) in the case of a homogeneous flow. For this case and for some elementary geometries, the added mass matrix is completely specified using potential flow theory ( $\rho=$ constant, $\mu=0$ ). The aim of this section is the validation of the numerical results obtained from the finite element code.

\subsection{Symmetrical section: rectangle}

First, a section with two perpendicular axes of symmetry, typically a rectangle of half length $a$ and half width $b$, is considered (Fig. 2). For this case, the corresponding added mass coefficients are given by (Blevins, 1995)

$$
m_{22}^{a}=\alpha \rho \pi a^{2} \text { and } m_{12}^{a}=m_{32}^{a}=0,
$$

where $\alpha$ is a parameter that is a function of $a / b$

The rectangle size $\left(a=7.5 \times 10^{-2} \mathrm{~m}, b=9.21 \times 10^{-3} \mathrm{~m}\right)$ is chosen as the approximation of the foil size which will be used later. Thus, the values of added mass coefficients should be close for these two geometries.

For this case, $a / b=8.143$, the parameter $\alpha$ can be approximated, according to table (14-1) given in Blevins (1995), by $\alpha=1.163$. Then, the added mass coefficients in water obtained from the potential flow theory are

$$
m_{22}^{a}=20552 \mathrm{~kg} / \mathrm{m} \text { and } m_{12}^{a}=m_{32}^{a}=0 .
$$

Eq. (16) is solved in the domain represented by Fig. 3. Fig. 4 shows the results depending on the meshing around a rectangle. As shown, an asymptotic value close to the theoretical value is obtained for about $8 \times 10^{5}$ nodes around the rectangle.

The added mass matrices (analytic and numeric methods) of the rectangle in the case of $\ddot{X}_{1} \neq 0, \ddot{X}_{2} \neq 0$ and $\ddot{X}_{3} \neq 0$ are given by

$$
\mathbf{M}_{\text {analytic }}^{(a)}=\left(\begin{array}{ccc}
0.555 & 0 & 0 \\
0 & 20.552 & 0 \\
0 & 0 & 1.46801 \times 10^{-2}
\end{array}\right)
$$

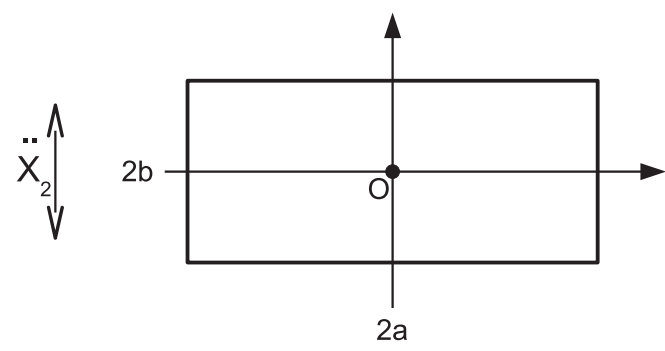

Fig. 2. Rectangle section. Acceleration direction denoted by $\leftrightarrow$ 


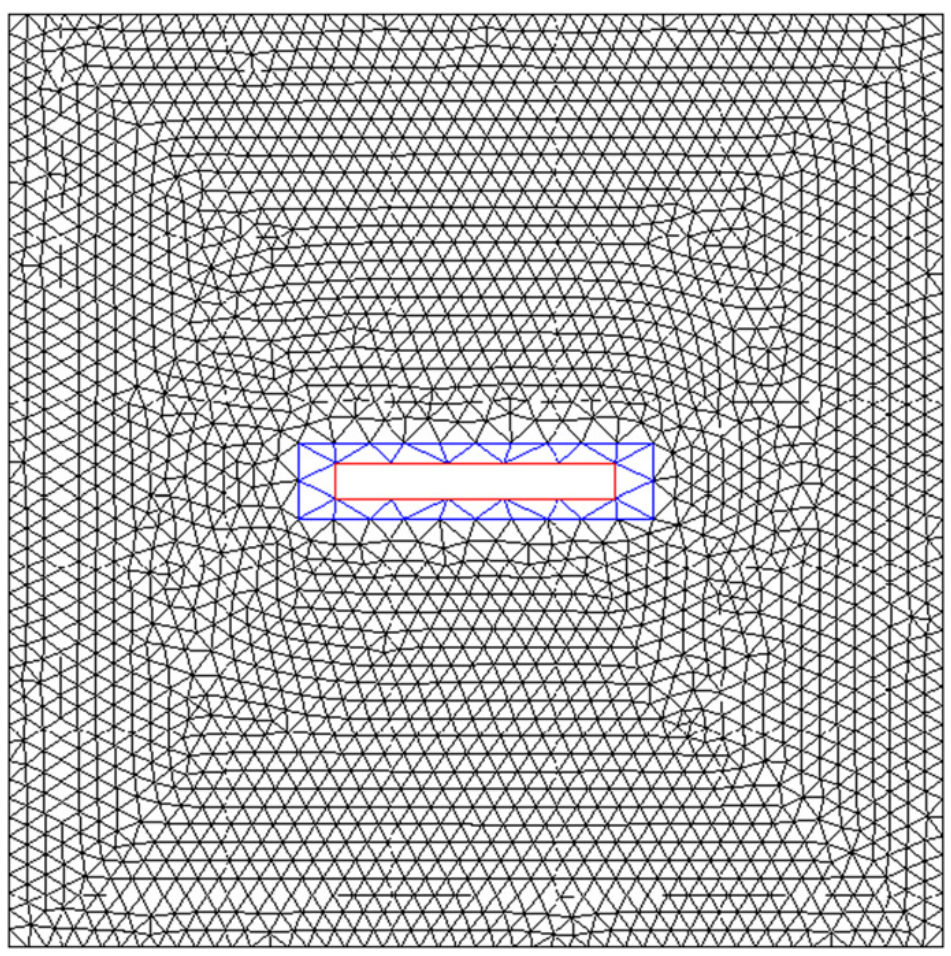

Fig. 3. Computational domain.
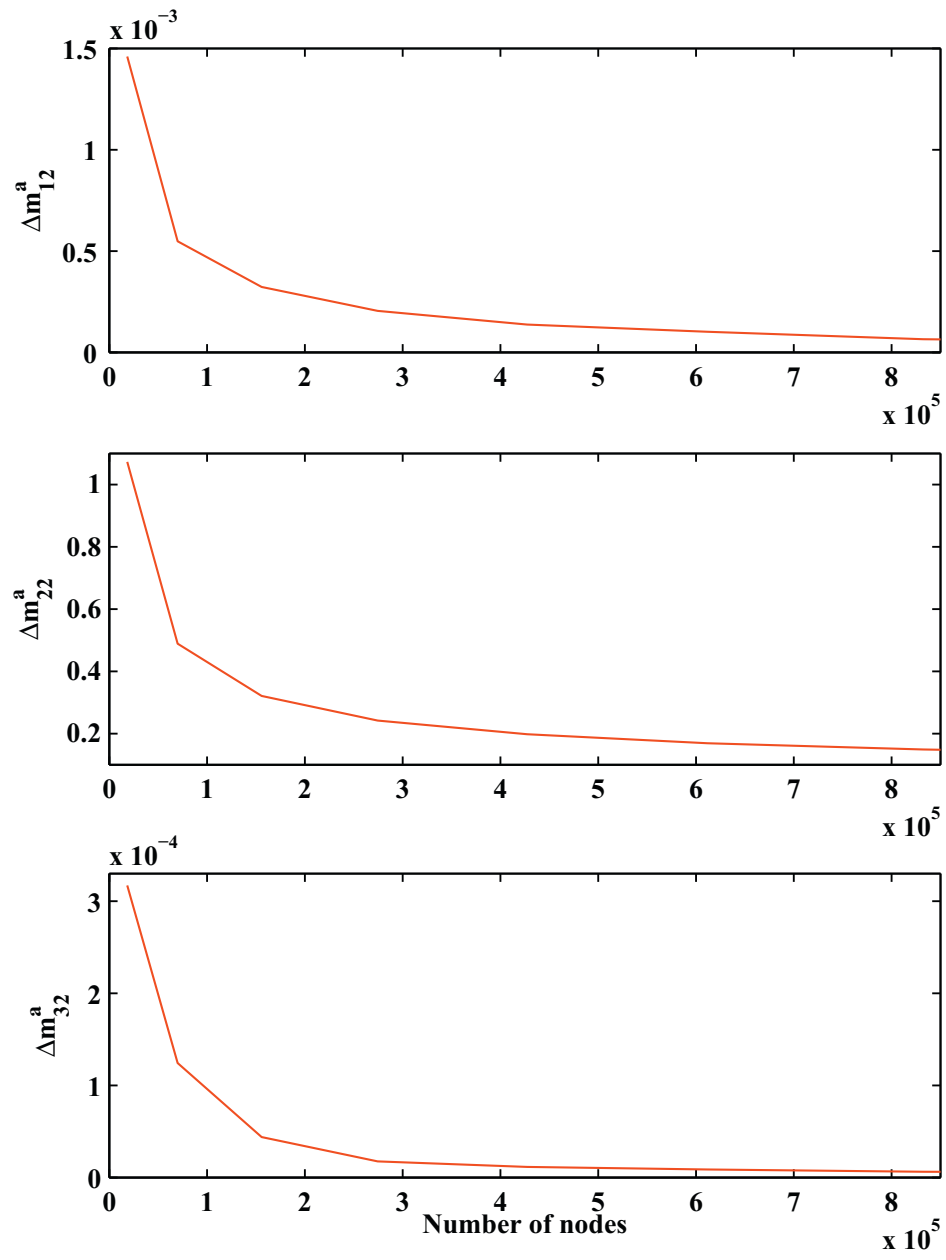

Fig. 4. Potential flow theory versus numerical results. 
and

$$
\mathbf{M}_{\text {numeric }}^{(a)}=\left(\begin{array}{ccc}
0.557 & -1.37 \times 10^{-4} & 2.39 \times 10^{-5} \\
-2.32 \times 10^{-4} & 20.354 & 1.28 \times 10^{-5} \\
2.64 \times 10^{-5} & 1.15 \times 10^{-5} & 1.41 .10^{-2}
\end{array}\right) .
$$

It is clear that the coefficients of the two matrices are very close. The numerical results are close to the analytical ones. The $0.96 \%$ of difference for $m_{22}^{a}$ and of $10^{-4}$ and $10^{-5}$ for $m_{12}^{a}$ and $m_{32}^{a}$ are obtained for about $4 \times 10^{5}$ nodes around the rectangle.

\subsection{Rotated symmetrical section: rectangle}

If the added mass matrix of a symmetrical section, about the coordinate axis, is known with respect to one coordinate system $(r, s)$ in an infinite fluid domain, the added mass matrix with respect to another coordinate system $(x, y)$ is rotated by an angle $\theta$ with respect to $r-s$ coordinate axis, as shown in Fig. 5, is given by (Blevins, 1995)

$$
\left\{\begin{array}{l}
m_{x x}^{a}\left(=m_{11}^{a}\right)=m_{r r}^{a} \cos ^{2} \theta+m_{s s}^{a} \sin ^{2} \theta, \\
m_{y y}^{a}\left(=m_{22}^{a}\right)=m_{r r}^{a} \sin ^{2} \theta+m_{s s}^{a} \cos ^{2} \theta, \\
m_{\beta \beta}^{a}\left(=m_{33}^{a}\right): \text { unchanged, } \\
m_{x y}^{a}\left(=m_{12}^{a}\right)=\frac{1}{2}\left(m_{r r}^{a}-m_{s s}^{a}\right) \sin 2 \theta, \\
m_{x \beta}^{a}\left(=m_{13}^{a}\right)=m_{y \beta}^{a}\left(=m_{23}^{a}\right)=0,
\end{array}\right.
$$

where $\beta$ denotes rotation about the origin of the $r$-s coordinate system. It is easy to note that, in this case the added mass matrix remains symmetrical. For $\theta=8^{\circ}$, the flowing added mass operator is obtained by using the above formulas:

$$
\mathbf{M}_{\theta, \text { analytic }}^{(a)}=\left(\begin{array}{ccc}
0.96704 & 2.7956 & 0 \\
2.7956 & 20.217 & 0 \\
0 & 0 & 1.46801 \times 10^{-2}
\end{array}\right),
$$

the flowing added mass matrix for the same configuration is obtained by using the numerical method

$$
\mathbf{M}_{\theta, \text { numeric }}^{(a)}=\left(\begin{array}{ccc}
0.96379 & 2.7267 & 3.89776 \times 10^{-4} \\
2.7333 & 20.069 & 4.30806 \times 10^{-4} \\
4.73461 \times 10^{-4} & 1.01870 \times 10^{-3} & 1.43026 \times 10^{-2}
\end{array}\right) .
$$

It is clear that the both matrices $\mathbf{M}_{\theta \text {,numeric }}^{(a)}$ and $\mathbf{M}_{\theta, \text { analytic }}^{(a)}$ are very close. It can be noted that the angle of attack makes the added mass matrix non-diagonal but remains symmetrical. For $\theta=8^{\circ}$, the surge added mass $m_{11}^{a}$ increased by $74.24 \%$, the heave added mass $m_{22}^{a}$ decreased by $1.63 \%$, the pitch $m_{33}^{a}$ was unchanged, the coupling term $m_{12}^{a}$ increased from 0 to 2.7956, while the other coupling terms remain null. The numerical results also show the same tendency. $m_{11}^{a}$ increased by $72.41 \% . m_{22}^{a}$ decreased by $0.82 \% . m_{33}^{a}$ remained almost unchanged with a gap of $0.676 \%$.

\subsection{Asymmetrical section: hydrofoil}

A NACA66312 type hydrofoil section is considered. It is the section already studied by Benaouicha et al. (2010). The center of rotation of the foil is taken at the point $O(0.25 \cdot c, 0)=(0.0375,0)$ as shown in Fig. 6 , where $c=0.15 \mathrm{~m}$ is the chord length. The corresponding added mass matrix is then obtained numerically by solving Eq. (16) in the domain represented

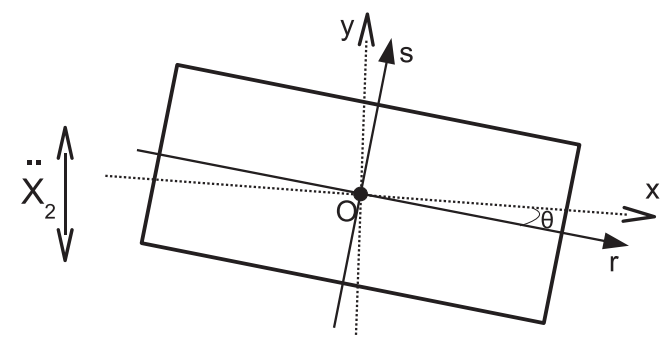

Fig. 5. Rotated rectangle section. Acceleration direction denoted by $\leftrightarrow$.

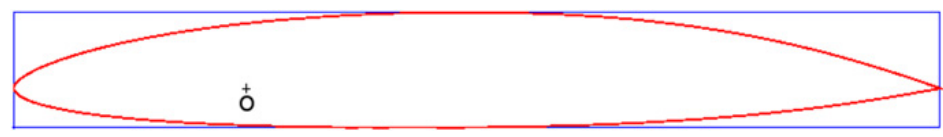

Fig. 6. NACA66312 type hydrofoil. 


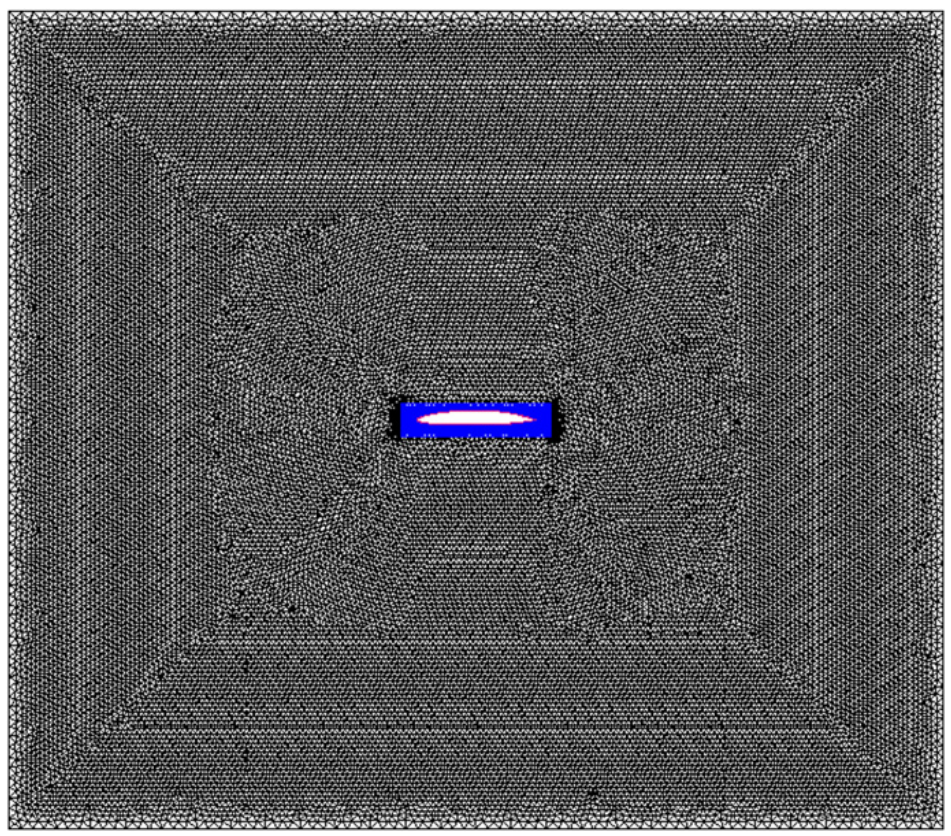

Fig. 7. Computational domain $\left(\theta=0^{\circ}\right)$.

by Fig. 7:

$$
\mathbf{M}_{\text {foil }}^{(a)}=\left(\begin{array}{ccc}
0.2606 & -1.49165 \times 10^{-2} & 1.1328 \times 10^{-2} \\
-1.4853 \times 10^{-2} & 17.059 & 0.63214 \\
1.13245 \times 10^{-2} & 0.63219 & 3.521 \times 10^{-2}
\end{array}\right) .
$$

The matrix is symmetrical but not diagonal because of the asymmetry of the foil.

\subsection{Rotated asymmetrical section: hydrofoil}

The formulas (24) are completed in the case of asymmetrical section, about the coordinate axis, as follows:

$$
\left\{\begin{array}{l}
m_{x x}^{a}\left(=m_{11}^{a}\right)=m_{r r}^{a} \cos ^{2} \theta+m_{s s}^{a} \sin ^{2} \theta-m_{r s}^{a} \sin 2 \theta, \\
m_{y y}^{a}\left(=m_{22}^{a}\right)=m_{r r}^{a} \sin ^{2} \theta+m_{s s}^{a} \cos ^{2} \theta+m_{r s}^{a} \sin 2 \theta, \\
m_{\beta \beta}^{a}\left(=m_{33}^{a}\right): \text { unchanged, } \\
m_{x y}^{a}\left(=m_{12}^{a}\right)=\frac{1}{2}\left(m_{r r}^{a}-m_{s s}^{a}\right) \sin 2 \theta+m_{r s}^{a} \cos 2 \theta, \\
m_{x \beta}^{a}\left(=m_{13}^{a}\right)=m_{r \beta}^{a} \cos \theta-m_{s \beta}^{a} \sin \theta, \\
m_{y \beta}^{a}\left(=m_{23}^{a}\right)=m_{r \beta}^{a} \sin \theta+m_{s \beta}^{a} \cos \theta .
\end{array}\right.
$$

In this case too, the matrix remains symmetrical. First, it has been determined by using a semi-analytical method. It consists to apply the analytical formulas (25) to a numerical result $\mathbf{M}_{\text {foil }}^{(a)}$. The following matrix is obtained for $\theta=8^{\circ}$ :

$$
\mathbf{M}_{\theta, \text { semi-analytic }}^{(a)}=\left(\begin{array}{ccc}
0.5819 & 2.3008 & 0.0992 \\
2.3009 & 16.7377 & 0.6244 \\
0.0992 & 0.6245 & 3.521 \times 10^{-2}
\end{array}\right)
$$

Second, a numerical method is applied by solving Eq. (16) in the domain represented by Fig. 8. The following added mass matrix is obtained:

$$
\mathbf{M}_{\theta, \text { numeric }}^{(a)}=\left(\begin{array}{ccc}
0.58245 & 2.3031 & 0.10145 \\
2.3032 & 16.752 & 0.63071 \\
0.10146 & 0.63075 & 3.58007 \times 10^{-2}
\end{array}\right)
$$

It is clear that the both matrices $\mathbf{M}_{\theta \text {,semi-analytic }}^{(a)}$ and $\mathbf{M}_{\theta \text {,numeric }}^{(a)}$ are very close. As in the case of symmetrical section, the added mass matrix remains symmetrical for an asymmetrical section with an angle of attack $\theta$. For $\theta=8^{\circ}, m_{11}^{a}$ increased by $123.29 \%, m_{22}^{a}$ decreased by $1.88 \%, m_{33}^{a}$ and $m_{23}^{a}$ remained unchanged, $m_{12}^{a}$ increased from 0 to 2.3 and $m_{13}^{a}$ increased from $1.13 \times 10^{-2}$ to 0.099 . The numerical results also show the same tendency. 


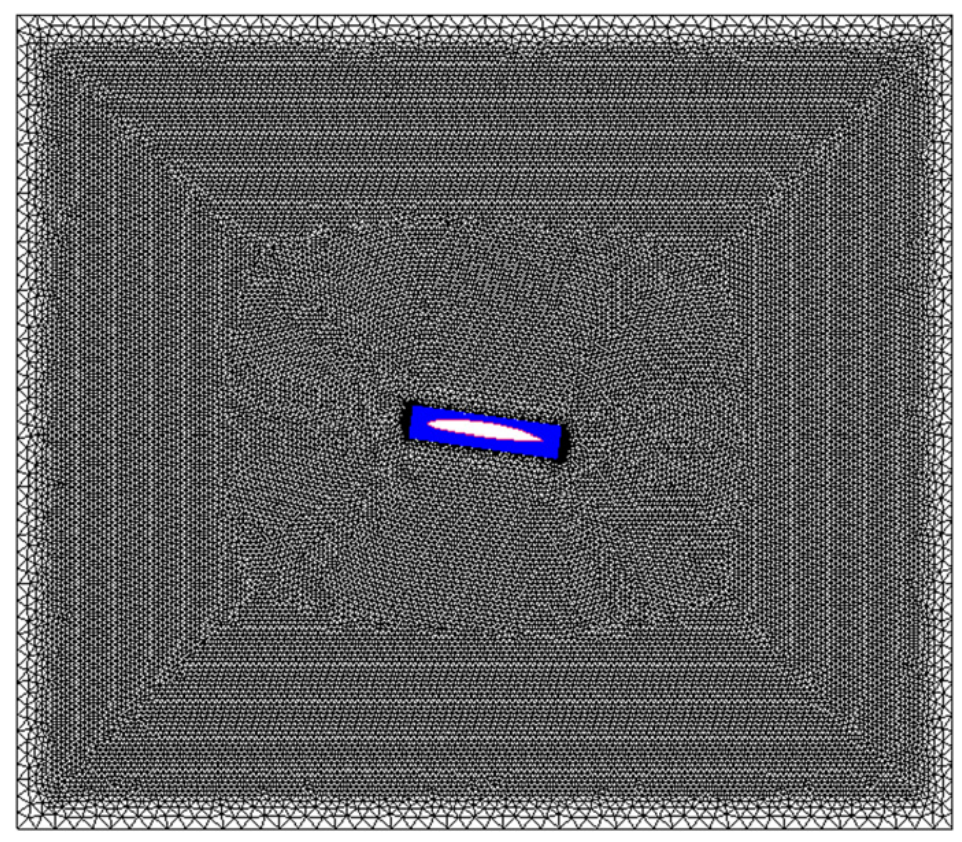

Fig. 8. Computational domain $\left(\theta=8^{\circ}\right)$.
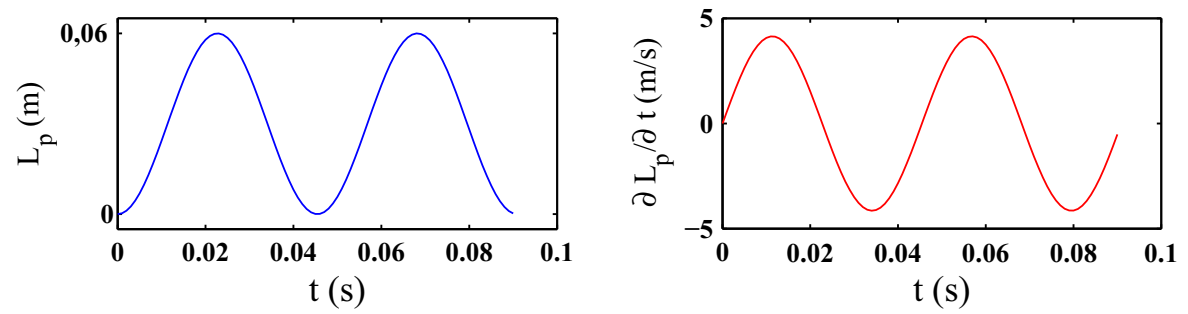

Fig. 9. Evolution of the cavity length $L_{p}(\mathrm{~m})$ and the rate of change $\partial L_{p} / \partial t(\mathrm{~m} / \mathrm{s})$.

\section{Numerical resolution in cavitating flow}

As it has been shown, the characteristic of a cavitating flow is that $\rho=\rho(x, y, t)$ is not constant over the foil surface. The method described in Section 2 computes the added mass and fluid density variation effects by solving Eqs. (16) and (17). In this section, the numerical solution of these equations are investigated for a simplified model of $\rho$, then for a fluid density coming from a numerical resolution of a cavitating flow (Benaouicha et al., 2009). Two cases of angle of attack are studied, $\theta=0^{\circ}$ and $\theta=8^{\circ}$.

\subsection{Simplified model of fluid density}

In the present problem (Eqs. (16) and (17)), the variations of $\rho$ are along the hydrofoil interface $\Gamma_{F S}$, namely in the $\mathbf{i}$ direction on the hydrofoil suction side. Let us consider the simplified model of an unsteady cavity of maximum length $L_{p_{\max }}$ oscillating at a frequency $f$,

$$
L_{p}(t)=\frac{L_{p_{\max }}}{2}(1-\cos (2 \pi f t))
$$

and the rate of change of the cavity is given by

$$
\frac{\partial L_{p}}{\partial t}=\pi f L_{p_{\max }} \sin (2 \pi f t)
$$

where $L_{p}(t)$ represents the cavity length at time $t(s), L_{p_{\max }}$ the maximum cavity length and $f$ the frequency of cavity oscillation. Fig. 9 shows the functions (26) and (27) during two periods and for $L_{p_{\max }} / c=0.4$, where $c$ is the chord length and $f=22 \mathrm{~Hz}$ (corresponding to experimental observations, Benaouicha et al., 2010).

Thus, $\rho(x, y, t)$ is defined for $(x, y) \in \Gamma_{F S}$ and $t \in \mathbf{R}^{+}$as

$$
\rho= \begin{cases}\rho_{v}=1 \mathrm{~kg} / \mathrm{m}^{3} & \text { if } 0 \leq x<L_{p}(t) \text { (vapor mass density), } \\ \rho_{l}=10^{3} \mathrm{~kg} / \mathrm{m}^{3} & \text { if } L_{p}(t) \leq x \leq c \text { (liquid mass density) }\end{cases}
$$


and

$$
\left\{\begin{array}{l}
\frac{\partial \rho}{\partial t}=\left(\rho_{v}-\rho_{l}\right) \delta\left(x-L_{p}(t)\right) \frac{\partial L_{p}(t)}{\partial t}, \\
0 \leq x \leq c, \quad(x, y) \in \Gamma_{\mathrm{FS}} \quad \text { and } \quad 0 \leq L_{p}(t) \leq L_{p_{\max }} .
\end{array}\right.
$$

$\delta$ being the Dirac function.

Fig. 10 shows the time evolution of the fluid density and the rate of change at $x / c=0.2$, on the hydrofoil.

\subsubsection{Added mass matrix coefficients}

The system of equations (16) is solved successively in the domains represented in Fig. 7 for $\theta=0^{\circ}$ and Fig. 8 for $\theta=8^{\circ}$. The variations of the density defined by the relations (28) are considered through the boundary conditions (16c). Figs. 11 and 13 (respectively Figs. 12 and 14) show the corresponding numerical results for $\theta=0^{\circ}$ (respectively $\theta=8^{\circ}$ ).

In Fig. 11 (respectively Fig. 12), the diagonal coefficients $\left(m_{11}^{a}(t), m_{22}^{a}(t), m_{33}^{a}(t)\right)$ of the added mass operator $\mathbf{M}^{(a)}(t)$ are represented for $\theta=0^{\circ}$ (respectively $\theta=8^{\circ}$ ). They are continuous functions of time and depend on the fluid density variations and then on the cavitation evolution. Indeed, it is shown that they are periodic functions with a period of $T=1 / f \approx 0.045 \mathrm{~s}$, where $f$ is the frequency of cavity oscillations. For both angles of attack $\left(0^{\circ}\right.$ and $\left.8^{\circ}\right)$, the coefficients $m_{11}^{a}(t)$ and $m_{22}^{a}(t)$ reach maximum values at the time for which the cavity length is minimal $\left(L_{p}=L_{p_{\min }}=0 \mathrm{~m}\right)$, and minimum
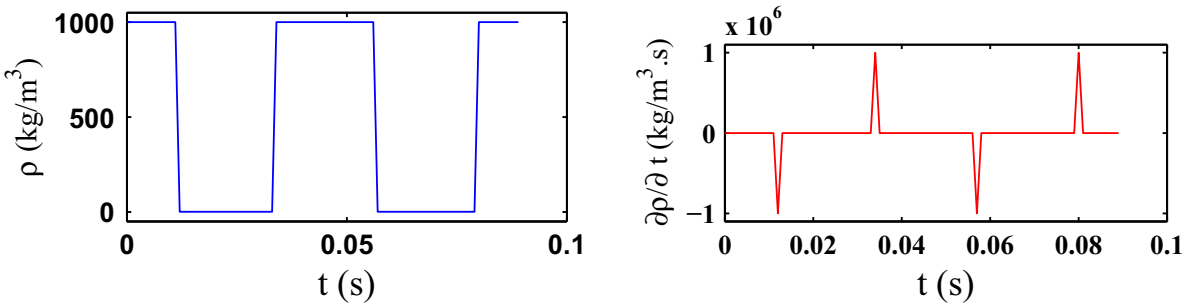

Fig. 10. Fluid density and rate of change versus time at $x / c=0.2$.
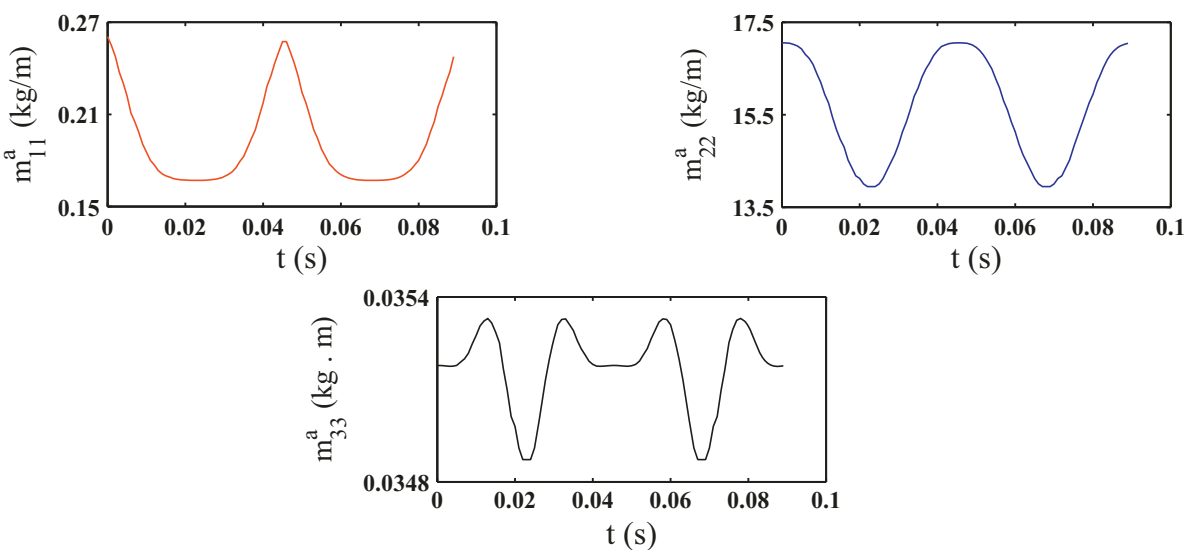

Fig. 11. Diagonal added mass matrix coefficients for $\theta=0^{\circ}$.

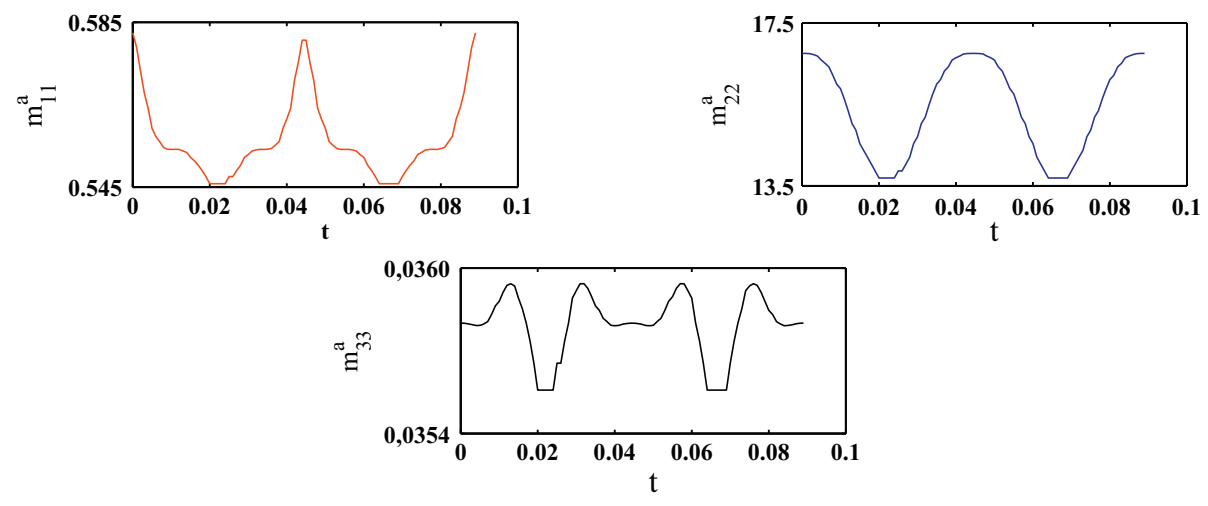

Fig. 12. Diagonal added mass matrix coefficients for $\theta=8^{\circ}$. 
values at the time for which the cavity length is maximal $\left(L_{p}=L_{p_{\max }}=0.4 \times c\right)$. As shown, the longitudinal (surge) added mass is two order of magnitude lower than the vertical (heave) one. The angle of attack $\theta$ increases the value of the surge added mass $m_{11}^{a}$ and decreases the value of $m_{22}^{a}$. The variations of pitch added mass $m_{33}^{a}(t)$ are more complex. They depend on the variations of the cavitation length $L_{p}$ and on the rate of change $\partial L_{p} / \partial t$. Indeed, this coefficient reaches a maximum value for the maximum rate of change, a minimal value for the maximum length of cavitation and an intermediate value for the minimum length of the cavity. $m_{33}^{a}$ is three order of magnitude lower than $m_{22}^{a}$. As expected, the angle of attack $\theta$ has no effect on the added moment $m_{33}^{a}$. For $L_{p_{\min }}=0 \mathrm{~m}$, the three coefficient values coincide with the results in the homogeneous flow case.

In Fig. 13 (respectively Fig. 14), the off-diagonal coefficients of the added mass operator $\mathbf{M}^{(a)}(t)$ are represented for $\theta=0^{\circ}$ (respectively $\theta=8^{\circ}$ ). It must be pointed out that the added mass operator is found to be not symmetrical. For $\theta=0^{\circ}$, the coefficients $m_{12}^{a}(t), m_{21}^{a}(t)$ and $m_{31}^{a}(t)$ have the same variation as the length of the cavity, whereas $m_{32}^{a}(t)$ has an opposite variations. The coefficients $m_{13}^{a}(t)$ and $m_{23}^{a}(t)$ depend both on the cavity length and its time derivative. The angle of attack $\theta$ has no effect on the added mass coefficients $m_{23}^{a}(t)$ and $m_{32}^{a}(t)$ but increases the values of $m_{12}^{a}(t), m_{21}^{a}(t), m_{13}^{a}(t)$ and $m_{31}^{a}(t)$ and modifies their time evolution. They are dependent on both the cavity length and its time derivative.

\subsubsection{Added damping matrix coefficients}

The system of equations (17) is solved for two cases of angle of attack: $\theta=0^{\circ}$ and $\theta=8^{\circ}$. The rate of variations of the fluid density defined by the relations (29) is considered in the boundary conditions.

The resulting coefficients of the added damping operator $\mathbf{D}^{(a)}(t)$ are shown in Figs. 15 and 17 for $\theta=0^{\circ}$ and in Figs. 16 and 18 for $\theta=8^{\circ}$. They are periodic functions with a same period, $T \approx 0.045 \mathrm{~s}$, than the cavity oscillations. They are null when the rate of change of the cavitation length $\partial L_{p} / \partial t$ is null.

It is thus clear that without variation of the fluid density at the fluid-structure interface, as in homogeneous flow, the added damping operator is null.

It is interesting to observe that $\left(d_{11}^{a}\right.$ and $d_{22}^{a}$ ) oscillate between positive and negative values conversely with the sign of $\partial L_{p} / \partial t$. $d_{11}^{a}$ exhibit two extrema and inflexion point at about $t=T / 2$. $d_{22}^{a}$ has quasi-sinusoidal shape and shows much greater values. $d_{11}^{a}$ is one order of magnitude lower than $d_{22}^{a}$. $d_{33}^{a}$ has a more complex shape with two oscillations during one period and is three order of magnitude lower than $d_{22}^{a}$.
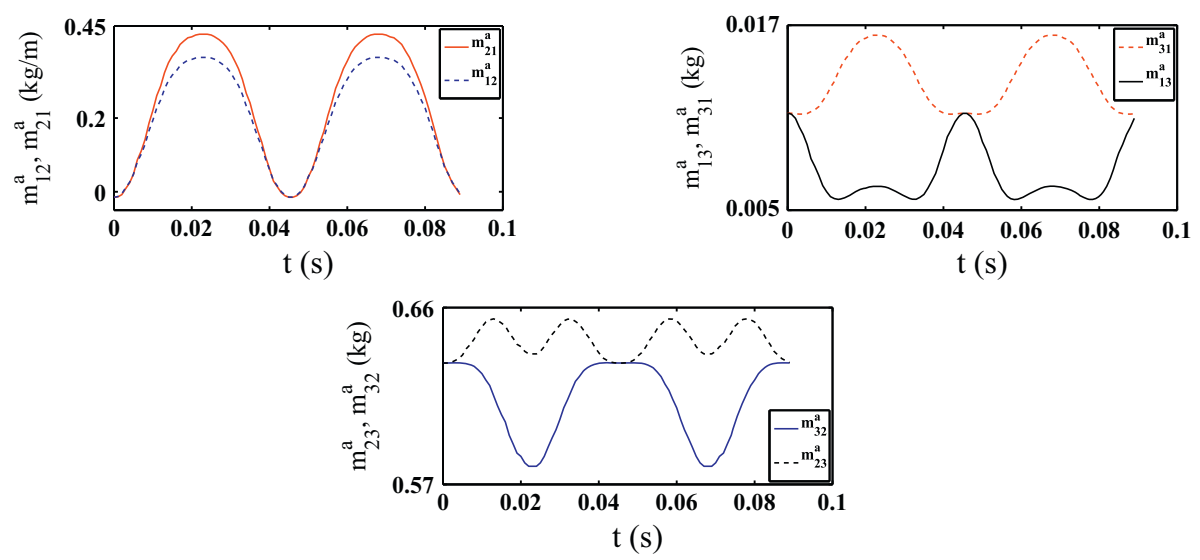

Fig. 13. Off-diagonal added mass coefficients for $\theta=0^{\circ}$.
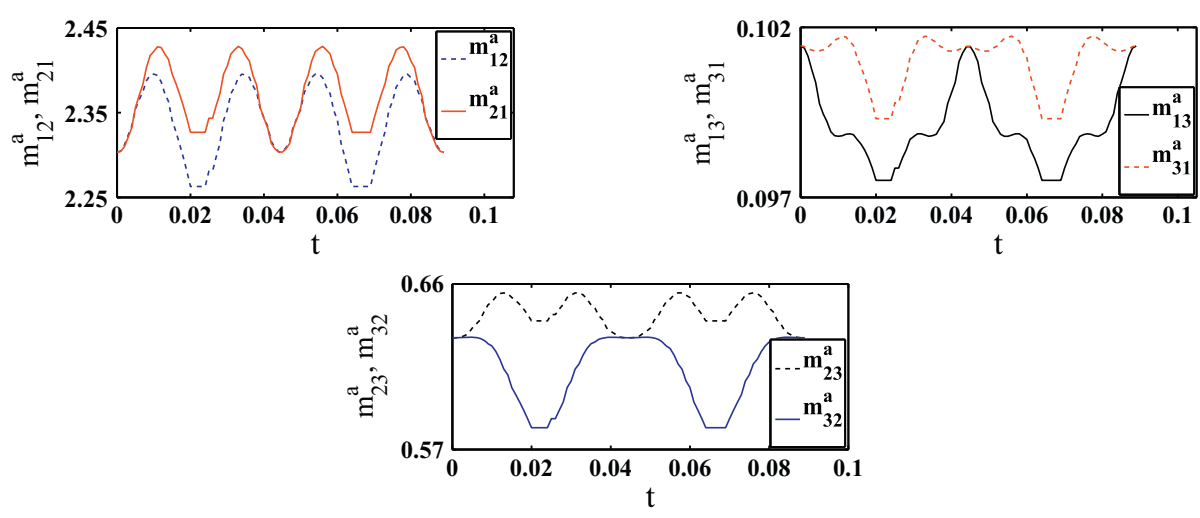

Fig. 14. Off-diagonal added mass coefficients for $\theta=8^{\circ}$. 

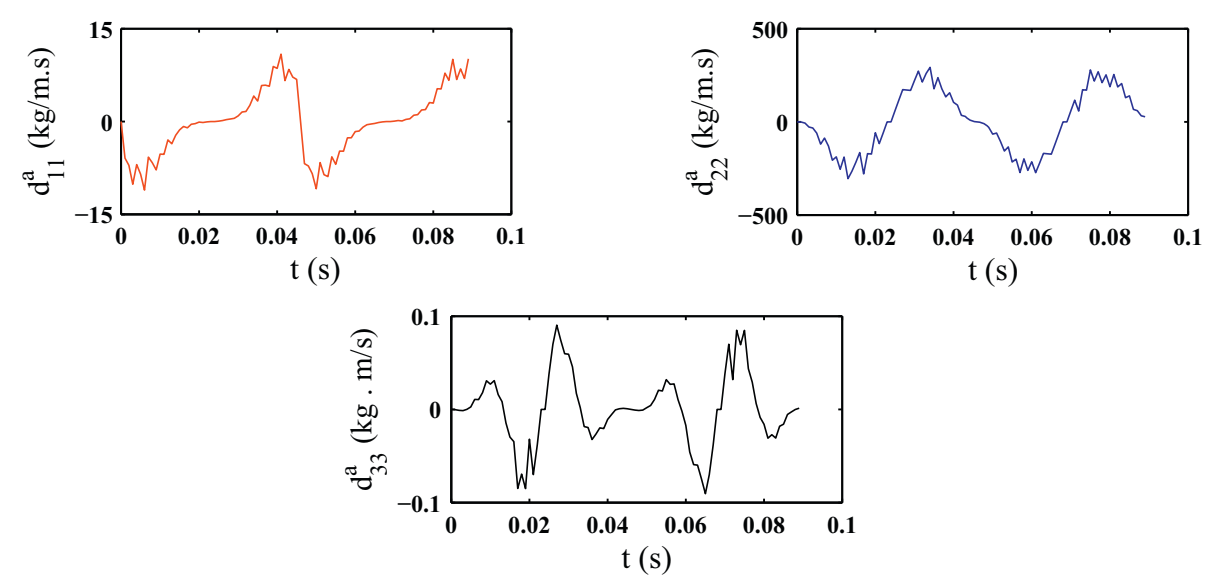

Fig. 15. Diagonal added damping matrix coefficients for $\theta=0^{\circ}$.
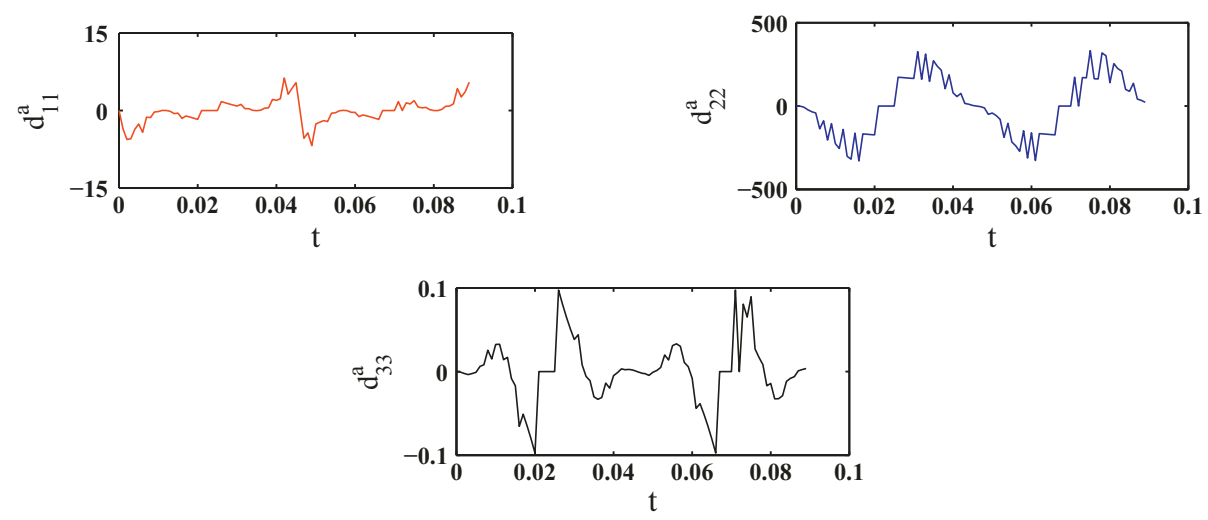

Fig. 16. Diagonal added damping matrix coefficients for $\theta=8^{\circ}$.
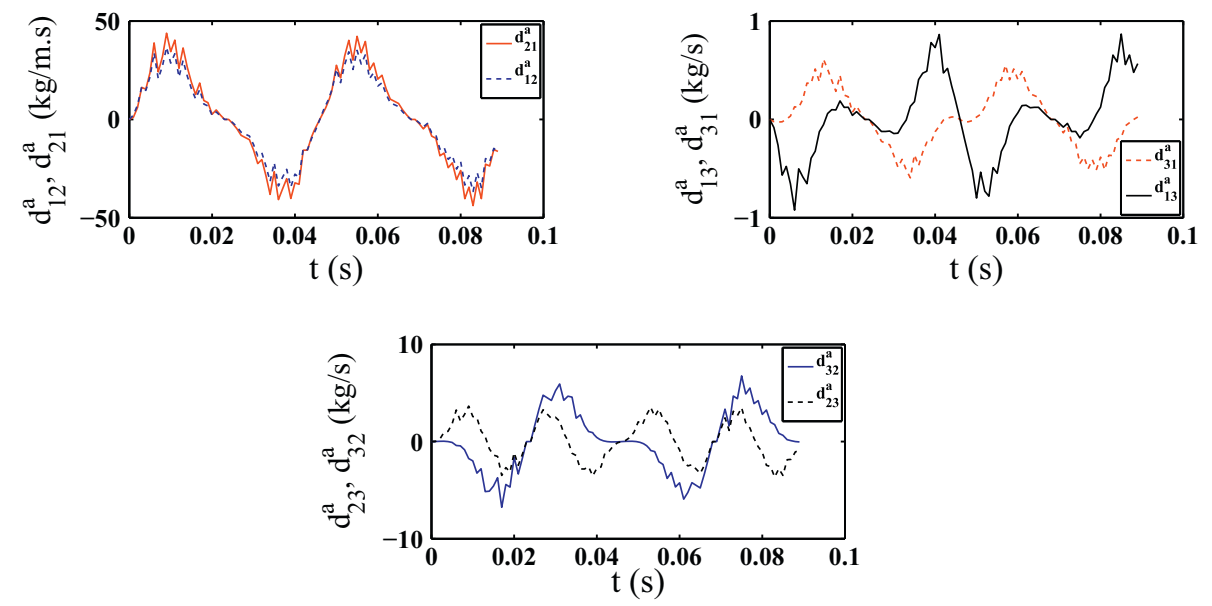

Fig. 17. Off-diagonal added damping matrix coefficients for $\theta=0^{\circ}$.

It can be noted (Fig. 17) that the operator is not symmetrical specially for $\left(d_{13}^{a}, d_{31}^{a}\right)$ and $\left(d_{23}^{a}, d_{32}^{a}\right) . d_{12}^{a}$ and $d_{21}^{a}$ have a quasi-sinusoidal shape and are very close.

It can be pointed out that the added damping coefficients oscillate between negative and positive values that can lead to a decrease or an increase of the total damping coefficient (in addition of structural and viscous dampings). This can have a strong impact on structure dynamics particularly concerning hydroelastic instability development.

The angle of attack $\theta$ has an effect only on the amplitudes of the added damping coefficients. The shape of the curves remains almost the same. 

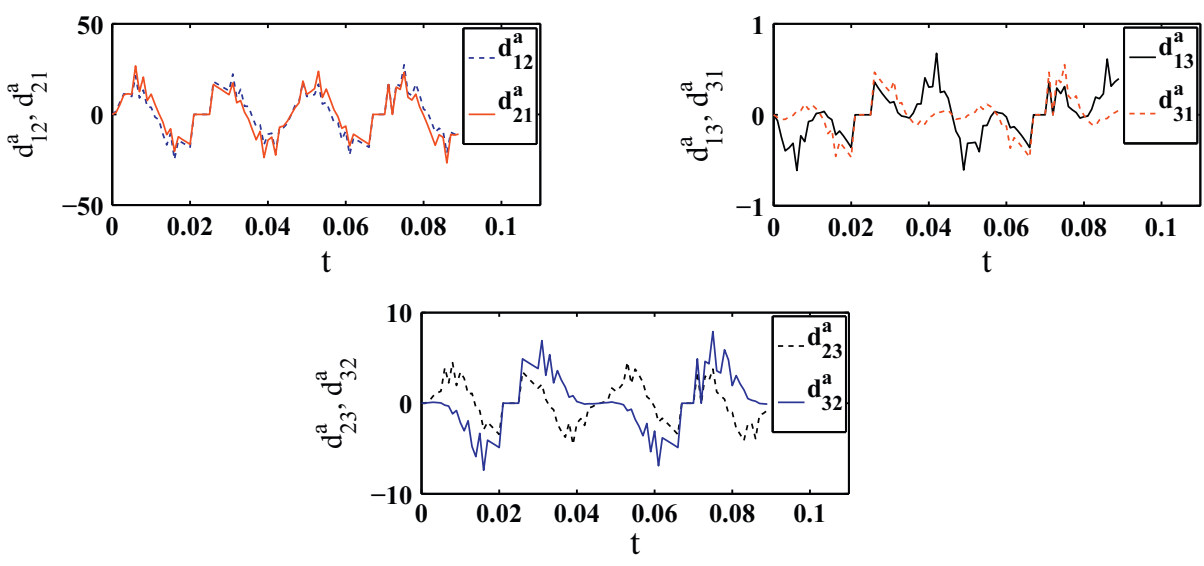

Fig. 18. Off-diagonal added damping matrix coefficients for $\theta=8^{\circ}$.

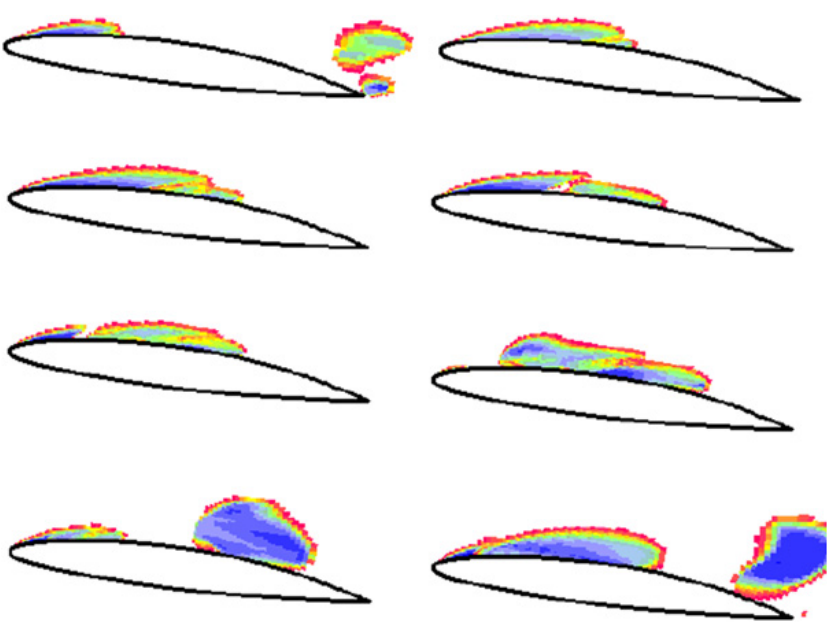

Fig. 19. Time evolution of the cavity shape (Frikha et al., 2008).

\subsection{Cavitating flow}

In this section, a more realistic space and time fluid density variations obtained from a numerical model of cavitation are introduced in Eqs. (16) and (17) used in the present model. RANS equations for a single phase flow are coupled with a barotropic state law that governs the density evolution according to the local pressure variation (Coutier-Delgosha et al., 2007).

Fig. 19 shows the numerical solution representing the oscillation of a cavity. The variation of fluid density as well as the rate of change along the fluid-structure interface can be deduced by

$$
\rho(x, y, t)=v(x, y, t) \rho_{v}+(1-v(x, y, t)) \rho_{l},
$$

where $\rho_{v}$ and $\rho_{l}$ are vapor and liquid mass densities respectively and $v(x, y, t)=V_{v} / V$ is the void fraction, $V$ is a volume of fluid cell and $V_{v}$ is a vapor volume in a fluid cell (Coutier-Delgosha et al., 2003). $v$ is a function of the cavity length.

Fig. 20 shows the fluid density variations versus time and rate of change at $x / c=0.2$.

Comparing to the sinusoidal idealized case (Eqs. (28) and (29)), it can be seen that the evolution of cavitation is more complex. It is periodic but not sinusoidal with a frequency of $f \approx 25 \mathrm{~Hz}$.

As shown, during the main oscillation, the cavity experiences (at $t \approx 0.021 \mathrm{~s}$ ) a secondary oscillation due to the generation of a secondary partial cavity development. It results in sharp peaks in density rate of change (Fig. 20).

\subsubsection{Added mass coefficients}

In this section, the problem (16) is solved by taking into account, through the boundary conditions, the fluid density variations resulting from the cavitating flow (Fig. 20). Two cases of angle of attack are studied: $\theta=0^{\circ}$ and $\theta=8^{\circ}$.

Fig. 21 (respectively Fig. 22) shows the diagonal coefficients of the added mass operator $\mathbf{M}^{(a)}(t)$ for $\theta=0^{\circ}$ (respectively $\theta=8^{\circ}$ ). For $\theta=0^{\circ}, m_{11}^{a}$ and $m_{22}^{a}$ exhibit a periodical behavior with a decrease of about $30 \%$ and $11 \%$ respectively when the cavity length is maximum. It is noted that $m_{11}^{a}$ is two order of magnitude lower than $m_{22}^{a}$. The secondary oscillation has no influence. A more complex feature is observed for $m_{33}^{a}$ with a successive increase and decrease of very small amplitudes 

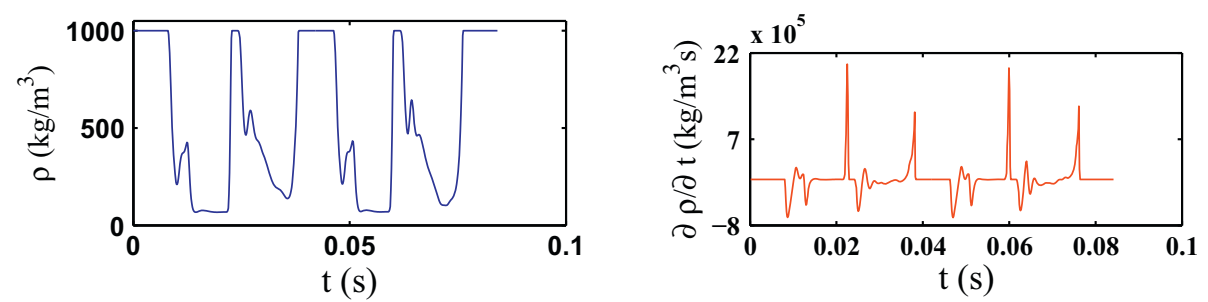

Fig. 20. Evolution and rate of change of fluid density at $x / c=0.2$.
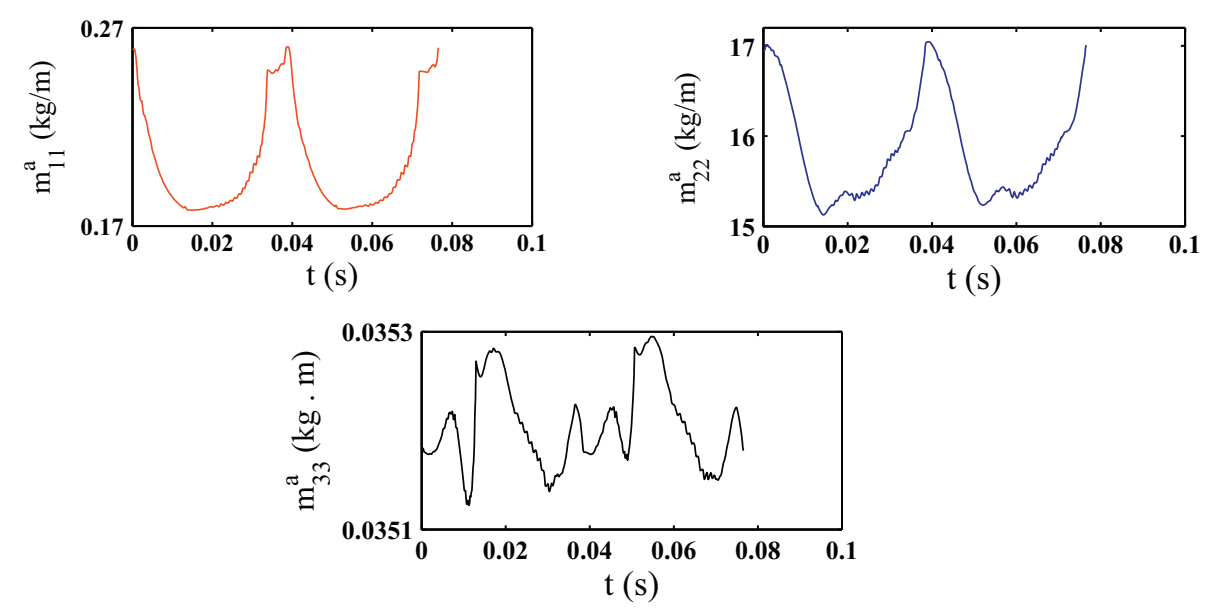

Fig. 21. Diagonal added mass coefficients for $\theta=0^{\circ}$.
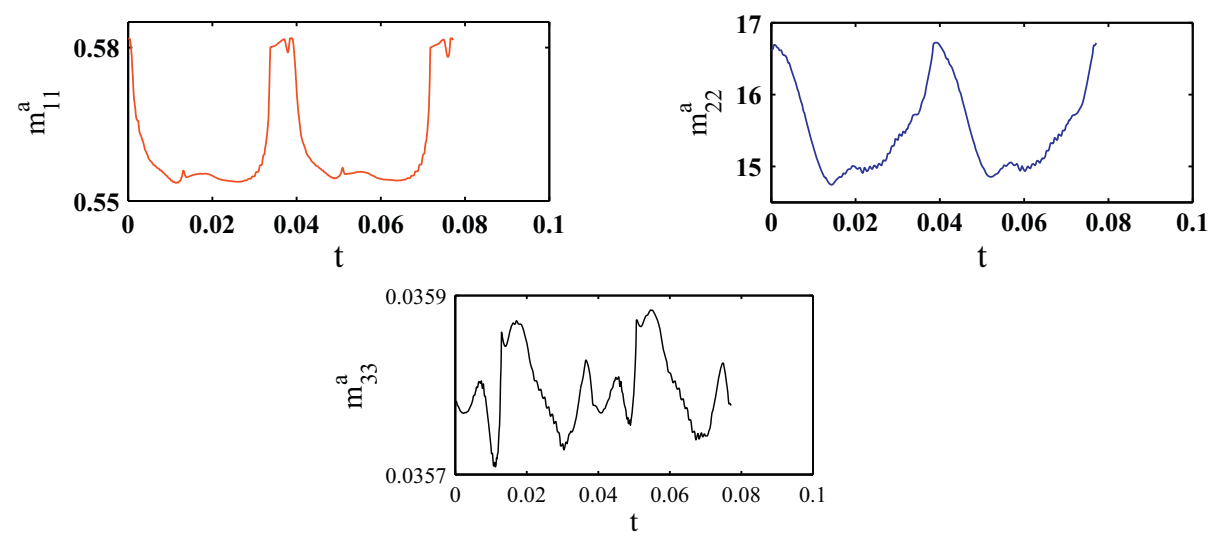

Fig. 22. Diagonal added mass coefficients for $\theta=8^{\circ}$.

(about $0.5 \%$ of the mean value) during the cavity oscillation. A stray increase is observed at the occurrence of the secondary oscillation. It observed that $m_{33}^{a}$ is three order of magnitude lower than $m_{22}^{a}$.

As expected, the angle of attack $\theta$ increases the value of the surge added mass $m_{11}^{a}$, decreases the value of $m_{22}^{a}$ and has no effect on the added moment $m_{33}^{a}$.

In Fig. 23 (respectively Fig. 24), the off-diagonal coefficients of the added mass operator are represented for $\theta=0^{\circ}$ (respectively $\theta=8^{\circ}$ ). They have approximately the same shape and orders of magnitude as in the case of a sinusoidal model of cavitation. Nevertheless, the variations are more complex in this case for which the model of cavitation is more realistic. It must be pointed out, once again, that the added mass operator is found to be not symmetrical. Moreover, it depends on the flow according to the density variations over the fluid-structure interface.

\subsubsection{Added damping matrix coefficients}

In this section, the problem (17) is solved by taking into account of the fluid density rate of change resulting from the CFD simulation (Fig. 20). Two cases of angle of attack are studied: $\theta=0^{\circ}$ and $\theta=8^{\circ}$.

Fig. 25 (respectively Fig. 26) shows diagonal coefficients of the added damping operator $\mathbf{D}^{(a)}(t)$ for $\theta=0^{\circ}$ (respectively $\theta=8^{\circ}$ ). They are periodic functions with a same period, $T \approx 0.04 \mathrm{~s}$, than the cavity oscillations. In this case, they have more complex shapes than those observed in the case of sinusoidal model of cavitation. This is due to the complex variations of 

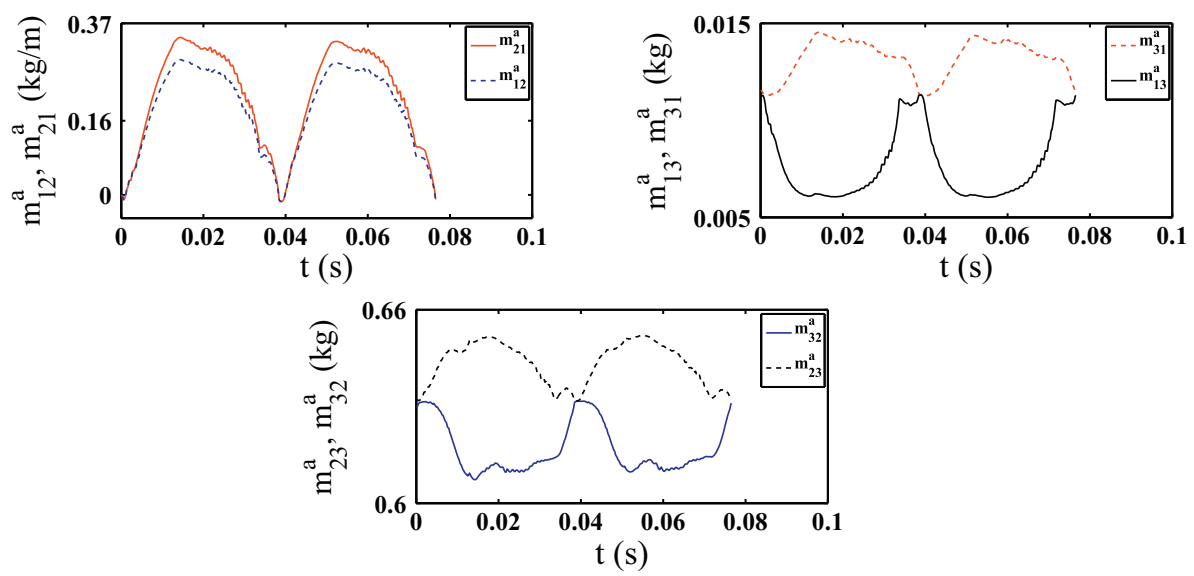

Fig. 23. Off-diagonal added mass coefficients for $\theta=0^{\circ}$.
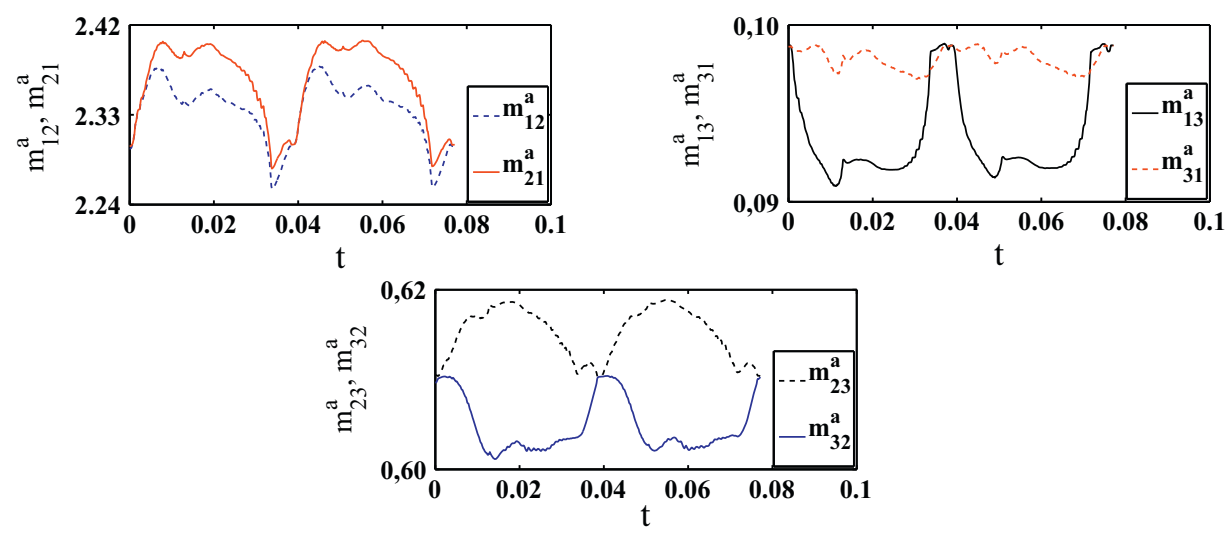

Fig. 24. Off-diagonal added mass coefficients for $\theta=8^{\circ}$.
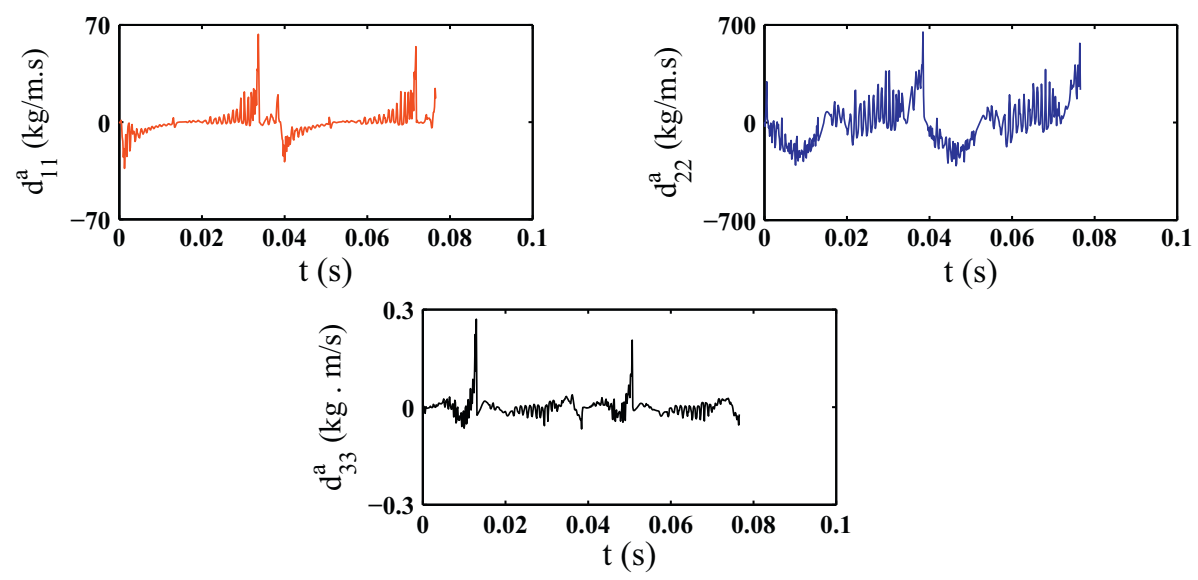

Fig. 25. Diagonal added damping matrix coefficients for $\theta=0^{\circ}$.

the fluid density over the fluid-structure interface (Fig. 20). $d_{11}^{a}$ exhibit two extrema and inflexion point at about $t=T / 2$. $d_{22}^{a}$ shows much larger values and it is one order of magnitude higher than $d_{11}^{a}$ and three order of magnitude higher than $d_{33}^{a}$.

Fig. 27 (respectively Fig. 28) shows off-diagonal coefficients of the added damping operator for $\theta=0^{\circ}$ (respectively $\theta=8^{\circ}$ ). It should be noted that the operator is not symmetrical, even if the coefficients $d_{12}^{a}$ and $d_{21}^{a}$ are very close. The quasi-sinusoidal shape observed in the case of the sinusoidal model of cavitation is replaced here by a more complex shape due to the more realistic model of cavitation. It can be observed that due to sudden sharp variations of the rate of change of the fluid density (Fig. 20), the added damping coefficients experience relatively high frequency oscillation contrary to the idealized sinusoidal evolution.

It can be observed again that the added damping coefficients are positive or negative. Negative added damping coefficients induce a decrease of the total damping that could be crucial for hydroelastic instabilities development. 

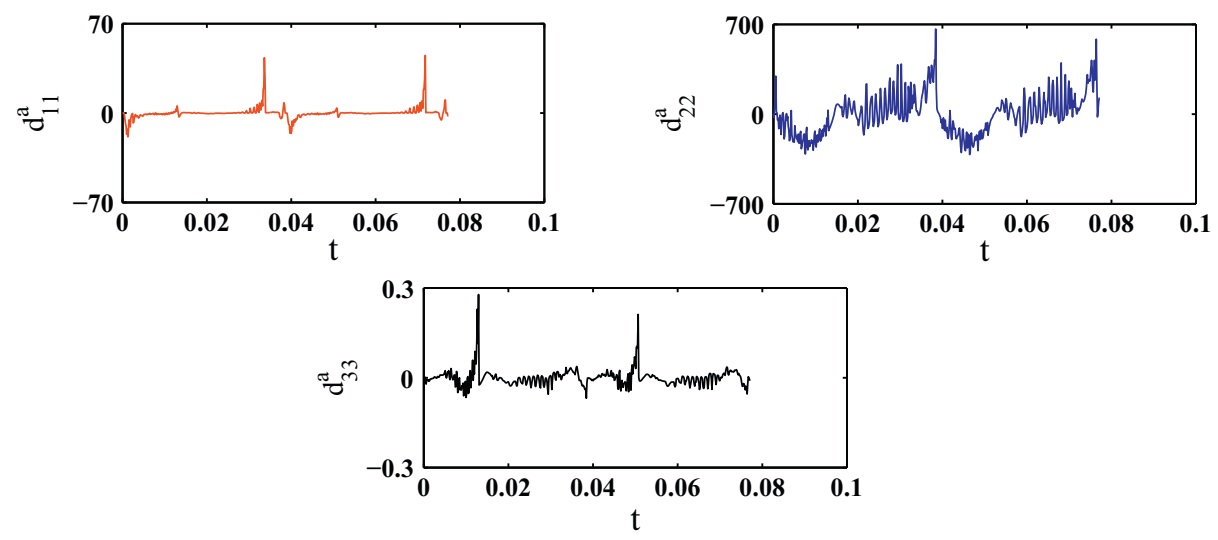

Fig. 26. Diagonal added damping matrix coefficients for $\theta=8^{\circ}$.

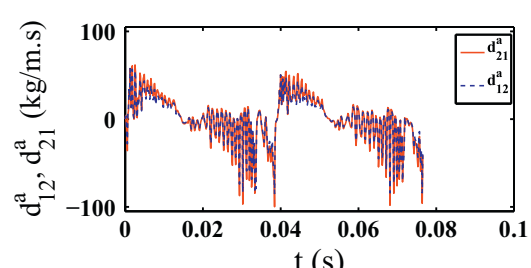

(s)
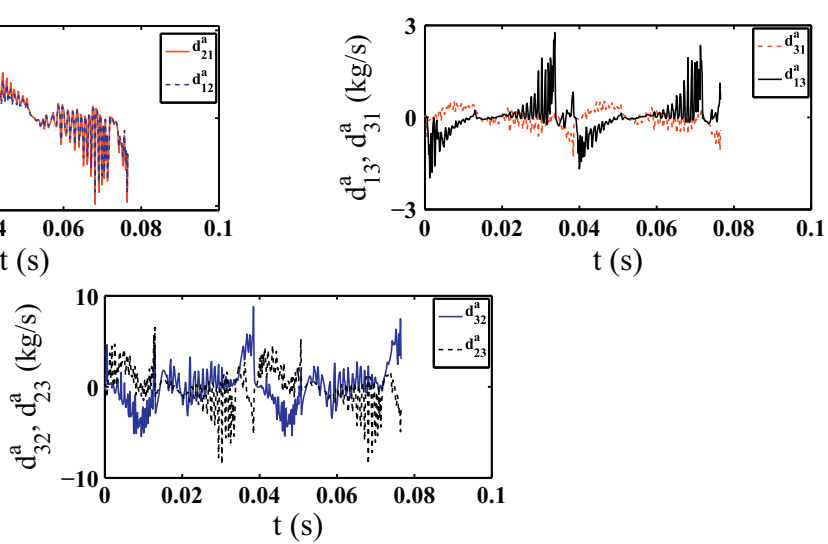

Fig. 27. Off-diagonal added damping matrix coefficients for $\theta=0^{\circ}$.
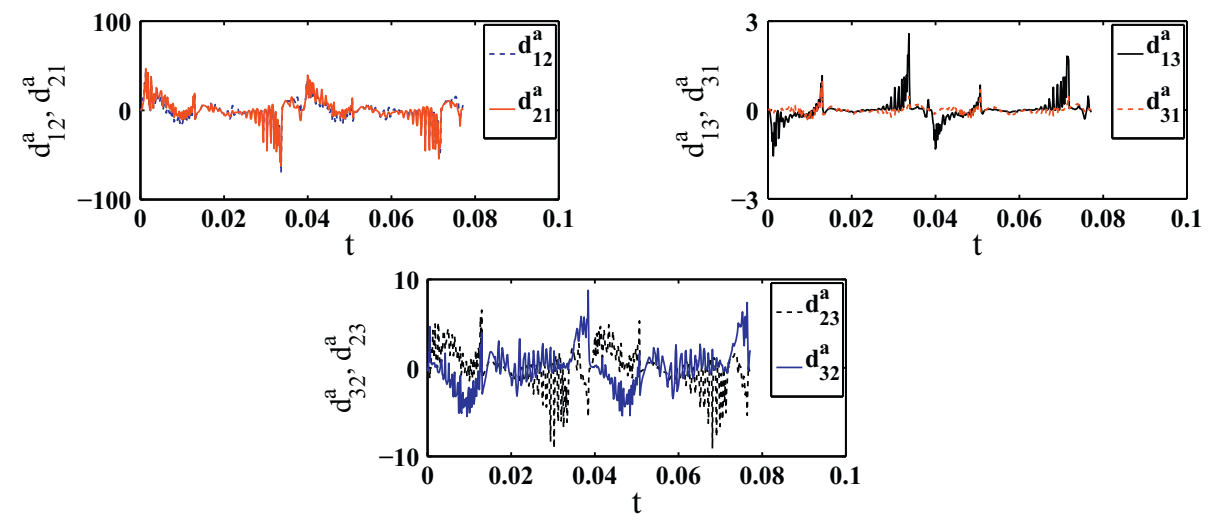

Fig. 28. Off-diagonal added damping matrix coefficients for $\theta=8^{\circ}$.

\section{Added mass effect on natural frequencies in cavitating flow}

Since the natural frequency of an elastic structure is inversely proportional to the square root of the mass of the structure, the effect of added mass on the $i$ th natural mode frequency $f_{i}$ can be expressed approximately as (Blevins, 1995)

$$
\frac{\left.f_{i}\right|_{\text {fluid }}}{\left.f_{i}\right|_{\text {vacuum }}}=\frac{1}{\left(1+\frac{m_{i}^{a}}{m}\right)^{1 / 2}},
$$

where $m$ is the mass per unit length of the considered structure (hydrofoil), $m_{i}^{a}$ is the added mass for mode $i$ and $\left.f_{i}\right|_{\text {fluid }}$ (resp. $\left.\left.f_{i}\right|_{\text {vacuum }}\right)$ the corresponding natural mode frequency (resp. in vacuum).

If we consider that the hydrofoil is made of stainless steel of density $7850 \mathrm{~kg} / \mathrm{m}^{3}$. The area of its cross section is $2.0369 \times 10^{-2} \mathrm{~m}^{2}$ and its mass per unit length is thus $m=15.9897 \mathrm{~kg} / \mathrm{m}$. The added mass coefficient, in not cavitating flow, 
that corresponds to the heave mode (in the $y$ direction) of the foil is $m_{22}^{a}=17.06 \mathrm{~kg} / \mathrm{m}$. Hence, the effect of the added mass on the corresponding mode frequency, in not cavitating flow, is given by

$$
\frac{\left.f_{2}\right|_{\text {fluid }}}{\left.f_{2}\right|_{\text {vacuum }}}=\frac{1}{\left(1+\frac{m_{22}^{a}}{m}\right)^{1 / 2}}=0.695
$$

The effect of the cavitation on the natural heave mode frequency $f_{2}$ can be evaluated by considering, in Eq. ( 32 ), the added mass coefficient $m_{22}^{a}$ in cavitating flow. It is found that $f_{2}$ is increased by about $3.1 \%$ for a cavity length of about $40 \%$ of the chord length.

\section{Conclusion}

The present study has analyzed theoretically some aspects of fluid-structure interaction phenomena, such as added mass effect, that could be expected to appear in cavitating flow developing over a vibrating structure. The cavitating flow considered herein is sheet cavitation that was found to occur at the leading edge of hydrofoils or blades and that develops partially along the structure. It is known that for given flow conditions, sheet cavitation has a large scale pulsating behavior that induces a strong space-time variation of the fluid density at the fluid-structure interface passing periodically from the vapor density (about unity) to the liquid density (about one thousand).

This point was examined through the derivation of Laplace equation written for the pressure and a new boundary condition at the fluid-structure interface that takes into account the rate of change of the fluid density that can be representative in a certain way of unsteady cavitating flow.

It has thus been found that because of fluid density variation, the boundary condition depends on both the acceleration and the velocity of the structure. Due to linearity, Laplace equation can be separated into two equations with two distinct sets of boundary conditions. The first one depends on the structure acceleration (inertial effect) and the second one depends on the structure velocity and the fluid density rate of change (damping effect). Laplace equation with the appropriate boundary conditions is then resolved for the two systems by the finite element method in homogeneous fluid and non-homogeneous fluid. For the latter, the fluid density variation at the fluid-structure interface is firstly represented by a simple sinusoidal model, then by a more realistic density variation obtained from a CFD resolution for cavitating flow.

Although the model is relatively simple, interesting general physical considerations can be drawn. Firstly, it has been found that for homogeneous fluid, the added mass operator is symmetrical and agrees very well with the results of classical potential theory showing that the method is a good generalization of the classical method and can easily be extended to any geometry.

In cavitating flow, it has been found that the added mass operator is not yet symmetrical. It depends on both the geometry and the flow. It has been found that the added mass operator coefficients oscillate as the cavity length oscillates. It decreases when the cavitation extends along the flow-structure interface. The decrease of the added mass coefficients induces an increase of the modal frequencies of the structure.

Finally, in cavitating flow the fluid density rate of change at the fluid-structure interface induces an added damping operator. It has been found that the coefficients of the added damping operator oscillate between positive or negative values according to the rate of change of fluid density. It can be pointed out that a negative damping could be critical for instabilities of dynamic systems. This point of critical importance, for operating systems in which cavitation develops, needs further analysis.

\section{References}

Ackermann, N.L., Asce, A.M., Arbhabhirama, A., 1964. Viscous and boundary effects on virtual mass. Journal of Engineering Mechanics Division 90, 123-130.

Amromin, E., Kovinskaya, S., 2000. Vibration of cavitating elastic wing in a periodically perturbed flow: excitation of subharmonics. Journal of Fluids and Structures 14, 735-751.

Axisa, F., Antunes, J., 2007. Modelling of Mechanical Systems. Fluid-Structure Interaction, vol. 3. Elsevier.

Benaouicha, M., Astolfi, J.-A., Ducoin, A., Frikha, S., Coutier-Delgosha, O., 2010. A numerical study of cavitation induced vibration. In: Proceedings of the ASME 2010 Pressure Vessels and Piping Conference (PVP2010), Bellevue, Washington, USA, July 18-22.

Benaouicha, M., Frikha, S., Ducoin, A., Astolfi, J., Coutier-Delgosha, O., 2009. Numerical study of hydrofoil deformation in a cavitating flow. In: Third International Congress Design and Modelling of Mechanical Systems, Hammamet, Tunisia, March 16-18.

Blevins, R.D., 1995. Formulas for Natural Frequency and Mode Shape. Krieger Publishing Company.

Brennen, C.E., 1995. Cavitation and Bubble Dynamics. Oxford University Press.

Caupin, F., Herbert, E., 2006. Cavitation in water: a review. Comptes Rendus Physique 7, 1000-1017.

CEA, 2009. CASTEM. French Atomic Energy Commission. URL $\langle$ http://www-cast3m.cea.fr $/\rangle$.

Chen, Y., Heister, S.D., 1995. Two-phase modeling of cavitated flows. Computers \& Fluids 24 (7), 799-809.

Coutier-Delgosha, O., Reboud, J., Delannoy, Y., 2003. Numerical simulations in unsteady cavitating flows. International Journal for Numerical Methods in Fluids 42 (5), 527-548.

Coutier-Delgosha, O., Stutz, B., Vabre, A., Legoupil, S., 2007. Analysis of cavitating flow structure by experimental and numerical investigations. Journal of Fluid Mechanics 578, 171-222.

Frikha, S., Coutier-Delgosha, O., Astolfi, J.-A., 2008. Influence of the cavitation model on the simulation of cloud cavitation on $2 \mathrm{~d}$ foil section. International Journal of Rotating Machinery, 12.

Goncalvès, E., 2011. Numerical study of unsteady turbulent cavitating flows. European Journal of Mechanics B/Fluids 30, 26-40. 
Han, R., Xu, H., 1996. A simple and accurate added mass model for hydrodynamic fluid-structure interaction analysis. Journal of The Franklin Institute 333B (6), 929-945.

Kopriva, E.J., Amromin, E.L., Arndt, R.E.A., Wosnik, M., Kovinskaya, S., 2007. High-performance partially cavitating hydrofoils. Journal of Ship Research 51 (December (4)), 313-325.

Korotkin, A.I., 2009. Added Masses of Ship Structures. Springer.

Kubota, A., Kato, H., Yamaguchi, H., 1992. A new modelling of cavitating flows: a numerical study of unsteady cavitation on a hydrofoil. Journal of Fluid Mechanics 240, 59-96.

Leroux, J., Coutier-Delgosha, O., Astolfi, J.-A., 2005. A joint experimental and numerical study of mechanisms associated to instability of partial cavitation on two-dimensional hydrofoil. Physics of Fluids 17, 052-101.

McConnell, K.G., Young, D.F., 1965. Added mass of a sphere in a bounded viscous fluid. Journal of Engineering Mechanics Division 91, $147-164$.

Newman, J.N., 1977. Marine Hydrodynamics. MIT Press, The Massachusetts Institute of Technology.

Ross, M.R., Felippa, C.A., Park, K.C., Sprague, M.A., 2008. Treatment of acoustic fluid-structure interaction by localized lagrange multipliers: formulation. Computer Methods in Applied Mechanics and Engineering 197, 3057-3079.

Ross, M.R., Sprague, M.A., Felippa, C.A., Park, K.C., 2009. Treatment of acoustic fluid-structure interaction by localized lagrange multipliers and comparison to alternative interface-coupling methods. Computer Methods in Applied Mechanics and Engineering 198, 986-1005.

Shukla, R., Pantano, C., Freund, J.B., 2010. An interface capturing method for the simulation of multi-phase compressible flows. Journal of Computational Physics 229, 7411-7439.

Ventikos, Y., Tzabiras, G., 2000. A numerical method for the simulation of steady and unsteady cavitating flows. Computers \& Fluids $29,63-88$.

Wang, G., Senocak, I., Shyy, W., Ikohagi, T., Cao, S., 2001. Dynamics of attached turbulent cavitating flows. Progress in Aerospace Sciences 37, 551-581.

Wang, X., 2008. In: Luo, A.C.J., Zaslavsky, G. (Eds.), Fundamentals of Fluid-Solid Interactions. Analytical and Computational Approaches, vol. 8. Elsevier.

Young, Y.L., 2007. Time-dependent hydroelastic analysis of cavitating propulsors. Journal of Fluids and Structures 23, 269-295.

Young, Y.L., 2008. Fluid-structure interaction analysis of flexible composite marine propellers. Journal of Fluids and Structures $24,799-818$ 